\title{
A search for explanation of the brain and learning: Elements of the psychonomic interface between psychology and neurophysiology II. Early behavior and its control, the origin of consciousness, and the rise of symbolic thought
}

\author{
PETER G. BURTON \\ University of Wollongong, Wollongong, New South Wales, Australia
}

\begin{abstract}
The psychonomic theory presented in the previous paper, which is based on a five-assumption cognitive framework of the brain's operation, is developed here into an account of the structured development of selective behavior generation. The central role of the motor transform is identified and a fundamental taxonomy of six successively early-learnable behavior classes is proposed as the basis of all higher human development, from infant behavior through cognitive and intentional behavior to symbolic thought. The basis of human performance is set out as broad-context encodings of experience that acquire attributes that selectively and automatically project into the action domain in each episodic percept-action cycle. The eyeblink is proposed as a normal flag of these cycles, which noradrenaline and dopamine control. Only the learned choice of consciousness in tervenes in this subconscious cycling: an account of the origin of consciousness emerges. Although it is devised to explain early personal learning, this theoretical scheme can be generalized to a sketch of the successive degrees of intellectual abstraction in the disciplines of our culture.
\end{abstract}

The aim of this work is to build upon the cognitive framework of the previous paper (Burton, 1990), to provide a systematic basis for understanding the exquisite variety of human behavior, and to understand how skills are developed.

The logical and cognitive model of the previous paper does not constitute a general explanation of the brain because it ignores 'internally generated' motor activity in ordinary behavior, the significance of which can be assessed from the psychologically negative affective connotations of contrived or enforced extended inhibition of motor activity. ${ }^{1}$ The purely logical learning model must be integrated into a wider context in which active involvement in learning is both recognized and comprehensible.

As in the previous paper, the focus is on early learning and behavior development, to provide the foundation elements of higher skilled behaviors. In particular, the logical basis of, and the neurophysiological substrates for, the control of human behavior is of prime concern. A taxonomy of primitive behavior types is developed, and, using the learning rules articulated in the previous paper, a plausible developmental progression through successively higher behaviors is outlined. This provides a basis for reflection on key conceptual issues of the brain and

Correspondence may be addressed to Peter G. Burton, Cognitive Science Group, University of Wollongong, Wollongong, NSW 2500, Australia.

-Accepted by previous editor, Lynn Nadel learning, including the theory of action generation, the significance of language, and the origin of consciousness.

In the theory of learning advanced in the previous paper, the registered perceptual influx is subconsciously 'processed' into temporally discrete episodes according to a threshold logic that requires significant information levels in each of several information channels (or groups of channels) to support a 'triangulation' logic for the progressive dissociative refinement of discerned perceptual features from the contextual background in which they occur.

'Objectification' of enduring or simply recurring polymodal segments of the episodically registered perceptual influx can be seen to arise epi-phenomenally in such a model via the dual requirements of the episodecomparison logic. The first element of this logic concerns 'mutual relevance' between the currently registered perceptual episode and a previously registered and stored 'indexable' episode. This in turn requires above-threshold similarity in the two episodes in the manner represented by a vector projection or vector dot product of the current episode onto the earlier stored episode-that is, active-information channels that characterized the stored episode must share some commonality with those of the current or proto-episode. The second element of this logic concerns the qualified episodic gating of the prelusory episode of perceptually registered information, which ensures that a current episode which indexes into the cortical termini of information channels with similar relative quantification over the active channels discounts the common elements of the prelusory episode. This is necessary be- 
cause a minimal degree of novel information (in terms of the information channels that are active or in terms of the relative quantification of information across active channels) in the content of any registered perceptual episode is required before the episodic gating signal-which initiates encoding of the episode-can be globally broadcast.

The combination of these two elements of the episodeprocessing logic affords the possibility of efficient use of memory in familiar environments by automatically ensuring that novel information in a familiar domain is acquired and stored distinct from, but in relation to, the common elements of the mutually relevant episode(s). As discussed in the previous paper, concomitant with articulation of new attributes from episode to episode, 'prototypes' are abstracted and successively consolidated from progressive encounters with familiar objects in the perceptual environment.

The feature-articulation process common to learning of objects of any type logically requires a recurrence of the object in an episode comprising a minimal degree of novelty, and at most one general feature can be articulated per recurrence. The 'object intension' progressively built up through such recurrences has a far greater richness than conventional representational ideas have admitted, because the automatic incorporation of the full polymodal perceptual context in which the object occurs is carried through successive episode storage. This is especially so of the earliest episodes supporting any object intension, although the dissociative learning mechanism admits ultimately of virtually - but never quite-complete dissociation of each object from its contextual background, provided of course that enough variation occurs across instances in the many episodes necessary to make this possible. The intensional, internally constructed object representation gains prominence as a familiar object from partial but progressive dissociation from its extensional episodic context.

Such character in the intensional 'storage' over declarative objects in memory permits an important generalization to be made concerning learning in the psychonomic framework. This is symbolized in Figure 1 in terms of the parallel development of the two complementary aspects of object learning that are supported by variation across a sequence of mutually relevant episodes. As the degree of (Aristotelian) abstraction of the object from its perceptual surroundings develops, so also must the complexity of the set of contexts in which the object has occurred. As the object itself becomes more and more generalized and free from particularities of any individual supporting episode, variety in the surrounding context supporting the 'minimal novelty of object' perspective of each successive episode must also increase.

In simple terms, the intensional storage model I have devised provides the basis of recognition of the object in an ever wider complex of contexts; ultimately, a familiar context itself may prompt a search for an object normally embedded in that context. A major goal of the present paper is to explain how such a 'reversal' becomes possible.

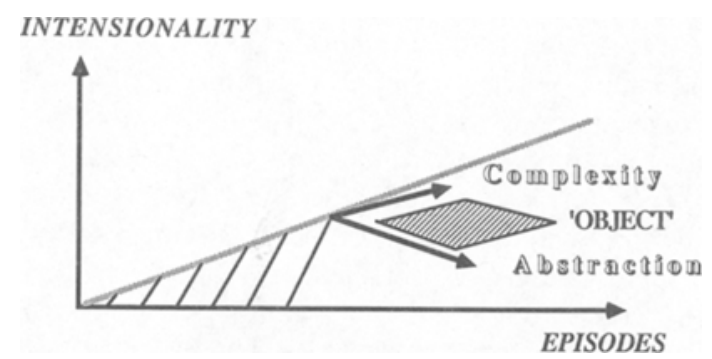

Figure 1. Dissociation and abstraction over complex experience. Dissociative learning begins with the separation of a perceptual object from its perceptual background, and progresses with each experience of the object from a new perspective by the articulation of attributes of the object from differences that are noticed between its successive presentations. Once the object has become 'known' and its features are familiar, additional experience yields the capacity to recognize the abstracted object in successively broader contexts.

The early learning that has been portrayed as rather automatic and passive for the learner, and typically dissociative and subconscious unless otherwise contrived, needs to be reconsidered in a more active light. However, the learner's initial unintentional actions can be distinguished from later intentional actions. This distinction is critical to the robustness of the learning framework, since we cannot require any particular order to the episodic learning sequence.

The chaotic, unpredictable perceptual environment that offers a unique individual learning trajectory for each of us is, in the episodic learning model, able to support similar quality of learning regarding the physical environment experienced no matter what actual episodic sequence is followed. Object learning merely requires recurrence with novelty; any extent of intervening experience unconnected with the object is permissible in the episodic learning model.

The randomness of early movements of each of us also provides a unique trajectory of learning, but ultimately there is quite a bit of commonality of acquired knowledge. Both passive-precognitive and active learning must be selective yet ultimately independent of the sequence of episodic experience; that is, learning must be stimulated by the content of the episode generating the recall of earlier relevant episodes, where the recall forms the basis of learning selectivity. All learning is present-experience driven.

Behavior will be considered in terms of the interplay among the three basic categories of object intension that can be known to the early learner. Summarizing from the previous paper, the early internal, experientially derived intensions are: (1) time slice, polymodal perceptual Type 1 objects, such as physical objects; (2) modally limited, temporally extended perceptual Type 2 objects, such as an external agent's spoken sentences or gestures; and (3) conceptual Type 1 objects, which first arise from the contrived attribution of disjoint labels to perceptually based objects, exemplified by explicit naming of objects 
from the physical environment in early language learning, and which eventually attain full two-way representational status between symbol and referent.

These categories must be broadened in two ways. First, Category 2 can be extended to admit kinaesthetically and proprioceptively specialized Type 2 objects derived from the learner's own internally generated movement patterns. This widens Category 2 explicitly from modal speciality in audition (heard utterance) or vision (seen gestures) to the kinaesthetic/proprioceptive sense, and thus generalizes Category 2 objects from more passively derived (e.g., audition) to more actively derived (e.g., sensed movements) objects. Because such objects were implicitly encompassed in our former use of the term 'Type 2 object'-since the kinaesthetic/proprioceptive sense is already incorporated in all our episodes as one of the six senses-in making this change we are really only highlighting this fact and not effecting a substantive extension.

Modally restricted Type 2 objects in audition were the subject of a brief discussion in the previous paper in terms of perceptual analysis of temporally extended, multiepisode objects. Even prior to gaining any experience in the analysis of temporally extended perceptual objects, such as sentences in audition or gestures in vision (where an enabling role of an external agent again suggests itself), we might admit the possibility of the learner becoming able to progressively discern, articulate, and refine attributes of perceptual Type 2 objects comprising personal proprioceptive and kinaesthetic perceptual registrations. The objectification of Type 2 objects spanning the kinaesthesis/proprioception modality requires a prior capacity to attend selectively to this modality, which in turn requires that this set of submodalities has become dissociable from the polysensory context of the perceptual influx to the central cognitive system (CCS) for each episode, by means of Type 1 learning.

If the early untutored capacity to process Type $2 \mathrm{ob}-$ jects is admitted, then in terms of our universal computational logic, the only possibility for Type 2 learning to occur is through a recurrence of the temporally extended kinaesthetic/proprioceptive object, which supports the necessary comparison-across-instances logic to provide articulation and refinement of such action sequences. In terms of this logic, nothing can be 'meaningful' about the execution of a totally novel percept-action sequence until features become articulated in the Type 2 object by means of the recurrence of a similar-but not identicalobject. Naturally, we recognize here the import of externally structured experience in facilitating the recurrence of similar but not identical extended experiences (e.g., in the parent 'encouraging' activation of pre-walking motor activity).

We can also recognize that the stepwise refinement of the activity sequence (with a focus on the complexity aspect in the temporal structure of the components to the activity) carries along with it the developing capacity to automate the activity sequence as a whole (thereby focusing on the abstract unity of the activity sequence). In these terms, the abstraction/complexity 'two-dimensional' growth of a Type 1 object intension via the recurrence of mutually relevant but distinct instances (Figure 1) is paralleled in the case of Type 2 objects by the twodimensional intensional growth in the automation-as-a whole/temporal-component-complexity dimensions. Thus, the general isomorphism between Type 1 and Type 2 objects is preserved, but this isomorphism now extends across all of the independent intensional objects of knowledge-that is, time-slice, polymodal perceptual Type 1 objects such as physical objects; modally limited, temporally extended perceptual Type 2 objects such as an external agent's spoken sentences or gestures; modally limited, temporally extended Type 2 motor objects representing internally constructed motor activities; and derived conceptual Type 1 or Type 2 object intensions arising from the resemblance across extensions of objects of the three primitive kinds and the symbolic labeling of these extensions.

In general, Category 2 objects now include any Type 2 objects regardless of modal salience or dominance, which, following the dissociative learning that enables discrimination of a small band of sensory submodalities from the full perceptual background, can be 'noticed' and followed selectively over an extended period on the episodic time scale.

Second, conceptual object intensions, which are initially derived by the contrivance of an external agent to 'meaningfully' link or associate disjoint objects, ${ }^{2}$ were portrayed in the previous paper mainly in the rather limited sense of denomination (i.e., in the appending of auditory names to objects in the physical environment). Although this provides elaboration of the internal object intension so named with a broader set of attributes, which in turn provide a richer basis for potential cross-reference between objects, this limited exemplification misses much of the breadth normally associated with the commonsense notion of a concept, of an 'idea.'

The required broadening of the concept intension category is illustrated by the parent's use of object-labeling strategies to bring two discrete object extensions under the same intensional label, just as we bring all the instances depicted in Figure 3 of the previous paper under the same intensional label (i.e., 'chair'). We progress from the naming of a particular instance of a chair object as "chair" [ $<$ this chair $>$ "chair"] to the generalized concept of $[<$ a chair $>]=[\{<$ this chair $>,<$ that chair $>\ldots\}$ 'chair']. The importance of this elaboration is that it facilitates recognition across time of different instances of the same concept by directing attention away from individual peculiarities of each chair to the salient common characteristics of chairs in general. The use of auditory labels that have little or no perceptual overlap with the other attributes of the named object is especially important in achieving concept broadening with minimal risk of confusion. ${ }^{3}$

It is this generalization of the notion of concept intension (i.e., the $[<$ the chair $>]$ to $[<\mathbf{a}$ chair $>]$ progres- 
sion) that provides a logical basis for denomination of recurring action segments with verbal nouns as action objects, just as Type 1 objects are denominated by nouns. That is, we can bring Type 2 objects explicitly into the labeling rubric.

With these broadened categories of knowledge all of which are acquirable through personal learning according to the one universal computational logic, we can now anticipate two further developments in knowledge acquisition.

First, in contrast with ordinary objects that 'grow' in intensionality through repeated but slightly changing cooccurrences of previously dissociated features, events are delimited temporally by abrupt changes in modal components of registered perception after a sequence of episodes. Event recall and object recognition become two different aspects of the Type 1 perceptual object-processing logic, being based on the logically distinct outcomes of episode comparison. This partially disjoint aspect of episode comparison, which supports the event/object distinction for perceptual object intensions, is later paralleled in the case of conceptual object intensions by the negation/ confirmation distinction in conceptual learning, which arises through communication and remains typicallybut not exclusively-socially mediated.

The second development is simply that any modally discrete Type 2 objects can exist contemporaneously, just as independent Type 1 objects can occur simultaneously in a single perceptual field. Voluntary activities that become automated and stereotyped through experience into habits of behavior (Dewey, 1922/1957) become important executable Type 2 objects, and these form the basis of our capacity to activate independent activities in coordination; habit formation is adaptively necessary to broaden our competence at simultaneous activity in different domains. The basic mechanism of habit formation needs clarification so that we can become more aware of this subtle and adaptively useful, if inexorable and sometimes surreptitious, process.

\section{THE ROLE OF BEHAVIOR IN LEARNING}

The context richness of episodic storage and the polymodality of the episodic gating proposed in the previous paper as the basis of declarative memory acquisition are prerequisite to the following proposals, as is the dissociative learning paradigm based on episode comparison. These notions were developed as cohesively as possible within the cognitive framework of the previous paper without any emphasis being placed on the active role of the learner in his or her development. Now a more integrative theory of action generation and learning can be woven.

Essential to the economy of this framework is the notion of learning as a primarily dissociative, contingent process of feature discovery, extraction, articulation, and attribution, which operates equivalently in learning about the static things of the environment and in learning over temporally extended perceptual objects. In the first case, learning involves the development of successively higher object intensionality, where the object is inherently context-rich and broadly polymodal (Type 1 object) with definite abstraction arising from recurring features of the object vis à vis its perceptual background, and articulated structure arising from the different perspectives (not purely visual) provided by the separate episodes that have given rise to the object intension over time. Learning of Type 2 objects, which begin with limited modal complexity (e.g., purely auditory, purely kinaesthetic, or purely visual as in gestures seen) but are typically prolonged in a smoothly modulated perceptual stream, likewise requires recurrence of the perceptual object for its 'existence' (i.e., for it to be noticed as an object) and higher articulated attributes to be acquired. ${ }^{4}$

The role of the learner's intentional or unintentional actions in the learning process is best conveyed by the concept of the motor transform, depicted schematically in Figure 2. Figure 2 schematizes the microstructure of a play segment involving a child reaching to touch an object and then, through an elementary motor transform, bringing the object into view. Here we have focused arbitrarily on the tactile sense in the initial episode and the visual and kinaesthetic/proprioceptive senses in the second, while admitting to each episode perceptual information captured from the concomitant auditory, taste/smell, and endoceptive senses.

The obvious utility of such a motor transform is to support the episode-comparison logic by the generation of changes and differences in the perceptual influx from episode to episode, thus helping to ensure a novel component in each successive episode. In the psychonomic theory, the achievement of a dissociable novel element to an episode, which is nevertheless relevant because of its commonality to an earlier episode, constitutes learning; the effective role of the motor transform is the facilitation of learning.

Now we need only one further step in the logic to have the beginnings of a theory of selective behavior generation. The exhibition of coherent behavior must be supported

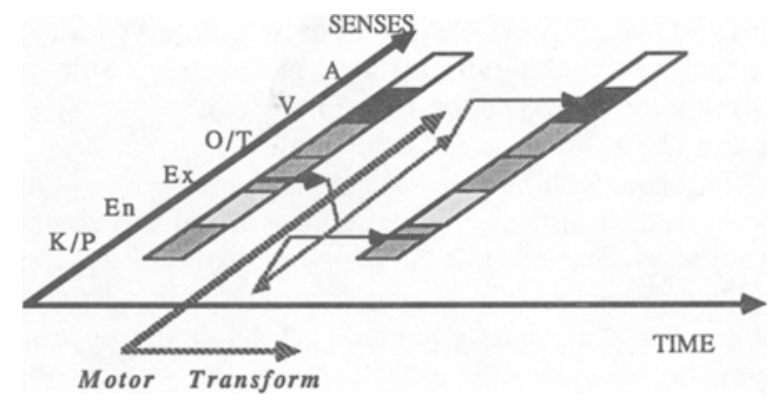

Figure 2. The motor transform. The purpose of the motor transform is the generation of differences between successive perceptually registered episodes. The example shown schematizes the generation of both movement and visual perception in response to touch, as in the removal from the mouth and inspection of some rough (or, were taste predominant, bitter) object. 
by the selective activation of motor processors. How does such selective behavior become possible? We need to relate the nature of any behavior generated to the content of the mutually relevant current and stored episodes, by way of the structure across channels in the overlap in the episode vectors reflecting each of the two episodes.

The motor transform provides the change in polymodal perspective that is the basis of the novel elements of each new episode that is required for learning to progress. The motor transform is, however, rather primitive in that it affords only the minimal necessary degree of novelty from episode to episode. But this is all that is needed; only gradual (but progressive) changes in the polymodal environment need be effected from episode to episode for learning to occur.

This portrayal of the general learning process fits well with observations of the detailed course of a child's play, with its rather steadily evolving character in each domain of play. Play segments-exemplary of our 'ticking-over' behavior-show smoothly modulated character even though switches of attention between segments produce a more chaotic overall appearance. After all, at most one attribute can be acquired per recurring relevant episode; too dramatic a change from episode to episode would act against progressive refinement of articulated attributes within a single domain. ${ }^{5}$

The basic thesis here is that the learning from each new perspective occurs whether or not the learner intends it. Whatever the basis for the motor transform, provided a new perspective is generated, learning is possible. In other words, an entirely chaotic and random activation of motor elements could serve to generate movements, and learning would still occur. Only the sequence of acquisition of attributes of objects is dependent on the actual motor transforms progressively invoked, and the sequence of attribute acquisition for any object is largely immaterial in early learning.

Early learning in the perceptual-motor domain can be, and is, largely unintentional and thus precognitive. It progresses without any need for conscious intervention, up to a certain point corresponding to the end of human infancy at 12-18 months of age, when language use begins. At which precise stage conscious intervention in learning becomes significant is of great interest and discovering the answer to this question will throw some light on the nature of consciousness itself.

Consonant with our present emphasis on the role of the motor domain in learning and on the origin and control of action generation, it is the temporal qualities of different categories of early exhibited behaviors that we now seek to articulate and systematize. A taxonomy of seven primitive behavior classes, which provide a reasonable basis for construction of all higher behaviors, is therefore the focus of the next section.

\section{A PSYCHONOMIC TAXONOMY OF PRIMITIVE BEHAVIOR CLASSES}

Even in the most straightforward and simplest of actions, many independently recognizable components are usually present. To simplify discussion, it is desirable to analyze behavior into its elementary components. Seven behavior classes are needed to capture the progressive augmentation of each individual's developing behavioral repertoire, to cover the progression from involitions to volitions, from volitions to intentions, and from intentions to goal-directed behavior of which 'thinking' is the epitome in both abstraction and level of competence.

It is the interplay among Type 1 objects, Type 2 objects, and higher order Type 1 and Type 2 concept intensions that we wish to articulate in the following taxonomy of behavior. We shall draw on our experience and observations of real-world learning in the process for better definition and exemplification of each behavior class. As the elementary classes of progressively acquirable behavior, of which higher human competent performance is comprised, we can identify: (1) primitive behavior, (2) coordinated behavior, (3) selected behavior, (4) skilled behavior, (5) concentration, and (6) deliberate behavior. All of these can be directly observed, whereas the last broad behavior class-(7) thinking-is especially mysterious because of its unobservability in others, which inhibits the normal comparison-across-instances logic by which one would otherwise compare and contrast one's thinking with that of another. By following a consistent strategy of search for the principle defining attribute which differentiates each higher behavior class from its precursor, it is hoped that some insight into the mysterious Category 7 will emerge.

\section{Class 1: Primitive Behavior}

Behavior in this category (schematized in Figure 3) summarizes the basic structure of perceptual learning, driven by the motor transform that interleaves between successive storable episodes of a play or action segment. The primitive percept-action cycle of behavior encompasses two subclasses of behavior: (1) reflex reaction to particular phylogenetically important percepts (reflexes, as in the suckling reflex or the dog's scratch reflex), and (2) involuntary, randomly initiated action elements.

Both the phylogenetically 'preprogrammed' capacity for selective movement that underlies reflex ${ }^{6}$ and similar early mimicry in humans ${ }^{7}$ and animals, ${ }^{8}$ and the capacity

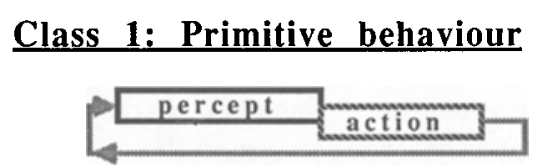

\section{Correlate: Instinctive S/R}

Figure 3. Class 1 behavior (instinctive $\mathbf{S} / \mathbf{R}$ ). Phylogenetically preprogrammed motor response to a particular direct perception. The preprogramming may operate at the level of simple, possibly random, action generation or the activation of a preprogrammed or Type 2 reflex activity sequence, as in sphexism. Such preprogramming implies the need for somatic evolution (Steele, 1981), since it is dependent on learning by an ancestor. The possibility for such preprogramming is opened up by the demonstration of the reprogamming of reflexes (Pavlov, 1955). 
for random initiation of movement, are necessary to encompass the instinctive behavior ${ }^{9}$-the early nonintended behavior-of all the animal kingdom. The importance of this latter capacity has often been overlooked.

In reality, the first class of primitive behavior forms a continuum between these two extremes, with apparently random-movement elements forming structured subgroups (e.g., early reaching movements), and with simple reflex movement (as in suckling) being but a rather direct example of a wider class of action including the capacity for mimicry of observed or heard actions.

The importance of the latter subclass of behaviors lies in its capacity to spawn a broad and randomized suite of percept-action links through 'accidental' recurrence of particular involitional motor elements in conjunction with particular, experientially recurring, perceptual contexts. This generates a rather chaotic repertoire of percept-action segments, which, together with the reflex repertoire, form the basis of the second class of behavior, which is learned from these elements.

\section{Class 2: Coordinated Behavior}

Coordinated behavior involves the simultaneous actuation of independent motor elements and movement units. Coordinated behavior is involved in broadening the scope of the action activated by a reflex prompt. It is also involved in bringing into replicable phase relationship independent motor or movement units that contribute to a unified but co-articulated response to a particular percept. For example, in replicating a heard phoneme string, the actual onset of actuation of independent sounds of the string will be highly overlapped, and learning to successfully mimic in production a heard polyphonemic sound involves considerable trial and error in the timing and phasing ('phrasing') of the necessary coordinated elements. Coordinated behavior is schematized in Figure 4.

The objectification of 'useful' coordination is merely a special case of general polymodal formation of an in-

\section{Class 2: Coordinated behaviour}

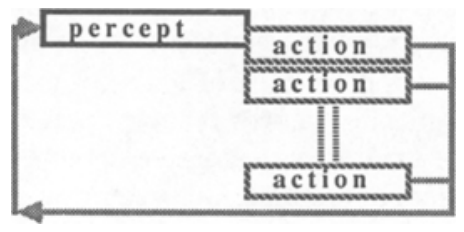

\section{Correlate: Learned coordination}

Figure 4. Class 2 behavior (coordinated behavior). Just as perceptual learning is possible in an unanticible environment, motor learning can arise from a random activation of motor elements. Kinaesthetic/proprioceptive perceptual learning based on selection and refinement of motor activity arises only by comparison across similar instances of coordinated action. 'Useful' coordinations can thus become 'objectified' irrespective of the sequence of enabling coordination of motor elements for any individual. ternal object intension. The object in coordinated movement is specialized to the kinaesthetic/proprioceptive sense, but it shares the general properties of all such Type 1 objects. In particular, learning and refinement of the coordination requires repetition for progressive modification or refinement.

Maximal flexibility and fluidity in movement is characterized by the conscription to use of a broad range of motor units, each operating within its normal dynamic range. The actuation of every relevant motor unit in every context would be bioenergetically excessive. Even actuation of all those formally relevant to a context each time would be excessive, because considerable redundancy exists, but establishment of a broad and pseudorandom repertoire of possible actions in response to a particular percept (i.e., context) provides the basis for extensive 'experimentation' of useful coordinations (Georgopolous, Schwarz, \& Kettner, 1986). Separate, possibly related, motor elements become coordinated in this way in a repertoire of independently accessible motor object intensions, called actions. This repertoire constitutes an array of action elements, some independent and some partially overlapping, and each action is represented formally by a vector of motorelement amplitudes that defines the 'shape' of the action when it is actuated.

\section{Class 3: Selected Behavior}

Actuation of a broad coordination of motor elements, some 'hard-wired' and some randomly activated, in an action linked to a particular global perceptual context provides the basis for Type 1 dissociative perceptual learning. Here the objects have, initially, a limited span over motor elements, each of which is an action vector that is highly salient in the limited modality of kinaesthesis/ proprioception.

Recurrences of coordinated action repertoires provide the basis for discovery and articulation of particular kinaesthetic and proprioceptive elements of movements that are regenerated in a recurring context. Learning in this category amounts to gaining control over selective recruitment of available motor units so that action that is 'appropriate' to each context can be selectively activated. This builds on the skills acquired in Class 2 behavior, where coordination and phasing control over motor elements develops, to provide the selection and/or emphasis of certain elements from the originally available, partially random coordination.

This objectification of coordinated motor elements as a coherent unit of movement in response to a global perceptual context, together with the increasingly selective (though not necessarily consciously selective, certainly not in the earliest stages) attention to salient features of the polymodal perceptual domain arising from increasingly focused perceptual learning, provides the basis of development of the selected behavioral repertoires of sensorimotor learning, as schematized in Figure 5.

Class 3, or sensorimotor, learning seems to be specifically associated with developing functionality of the pari- 


\section{Class 3: Selected behaviour}

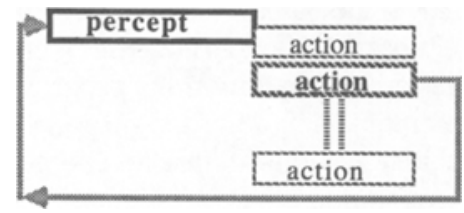

\section{Correlate: Response repertoire, requiring dissociation and selection}

Figure 5. Class 3 hehavior (selected action). Progressive kinaesthetic/proprioceptive modality learning leads to differentiation of alternative actions that can be selectively activated in response to a particular attended perception. The selective linking of an action to a particular percept logically requires prior learning in both the perceptual and the motor domain. In the perceptual domain, dissociation of perceptual features from the ever-changing perceptual influx must be possible, whereas in the motor domain, selection of specific motor combinations that incorporate tuned' relative amplitude motor elements must have been ohjectified from the initially random repertoire of motor coordinations.

etal association cortex of the brain. ${ }^{10}$ Capture of this level of behavior by neural networks has been exemplified in reading in Rosenberg and Sejnowski's (1986) NetTalk. This level or class of behavior seems particularly amenable to modeling with a connectionist 'parallel distributed processing' paradigm (Rumelhart \& McClelland, 1986), where variable recurrences of percept and action can lead to a strengthening in the developing programmed connection between one particular perceptual element (or group of elements) and one particular action element (or group of elements).

Appearance of Class 3 behavior leads to a progressively refined programming of a selected response to a selected stimulus. Learning mechanisms operate essentially dissociatively, both in the general sensing domain and in the motor domain of action, to provide naturally increasingly selectivity in behavior. Even so, the randomness of the perceptual environment (the 'large, blooming buzzing confusion') and the randomness of the arbitrarily activated ('random') early motor domain together rapidly provide the basis for a very broad range of Class 3 behaviors that can be learned via this double-selection, double-refinement process. The primitive percept-action cycle has become refined, and by the end of the first year, many regular behaviors appropriate to context are exhibited by the human infant.

Class 3 behavior and habit formation. Class 3 behavior epitomizes our normal everyday, unproblematic ticking-over mode of behavior, in which we follow an unpreoccupied meander through the ordinary perceptual environment, not being too surprised by anything and never being at a loss for something to do. This class of learning, involving cycling from increasingly selectable attention to increasingly selectable action to progressively encompass a wider range of capacity, is in fact the predominant class of our entire behavioral repertoire and occupies most of our waking-and perhaps also our sleeping-hours.

The range of our perceptual submodalities is very large across each sensory modality, and 'percept' can range across all of these submodalities in each of the six senses. Likewise, the breadth of possible selected action response is huge given the large number of individual motor units available for early programming into coordinations (Rothwell, 1987). One might imagine no limit to the variety and complexity of replicable behaviors that might be developed with increasing experience of Class 3 behavioral learning - that is, active sensorimotor learning.

There is, however, one major limitation that arises over time from this doubly dissociative learning paradigm. The problem arises in two parts. On the one hand, perceptual learning progressively narrows the scope of 'interesting' perceptually salient features of the environment. On the other hand, an equivalent dissociative learning operates to progressively select from an initially broad action element repertoire a smaller and smaller subset for 'routine' actuation. The early period of random activation of motor elements gives way to more stable and selective utilization of the motor system. In ordinary terms, this amounts to the progressive stereotyping of exhibited behavior.

Increasing stereotypy in thought and decision making is a corollary to what happens in bodily use over time. Increasing psychological resistance ('neophobia') to an uncontrollably changeable perceptual environment increasingly selects from the possible range an ever smaller interesting subrange of percepts to which a response is generated. The responses themselves become progressively refined and stereotyped so that the normally activated percept-action links become, in the routine of everyday activities, fewer and fewer.

Through inactivity, percept-action links that had been developed through recurrence in early learning begin to wane in strength and eventually become 'dormant.' In this way, whole segments of motor units that had been generally available become progressively, but surreptitiously, unavailable. Only by the deliberate reintroduction of a stochastic element to our behavior (such as a change to a new environment by relocation) or the deliberate reintroduction of overtly novel movement coordinations (such as a new sport or activity) can, by means of the establishment of new or renewed percept-action links, interrupt the otherwise inexorable decline into a progressively dormant percept-action system, with the available remnants declining in number but subject to increasing frequency of use.

Stereotypy and habit are adaptively valuable in the early learning of selective behavioral competence, in enhancing the likelihood of increasing refinement through recurrence of selected behaviors, and in enabling sufficient recurrence of these behaviors so that even complex responses to particular percepts may become, ultimately, routine and automated. However, it is the subconscious aspect to the dissociative learning involved that ultimately provides the risk of inexorable-and subliminal-degradation 


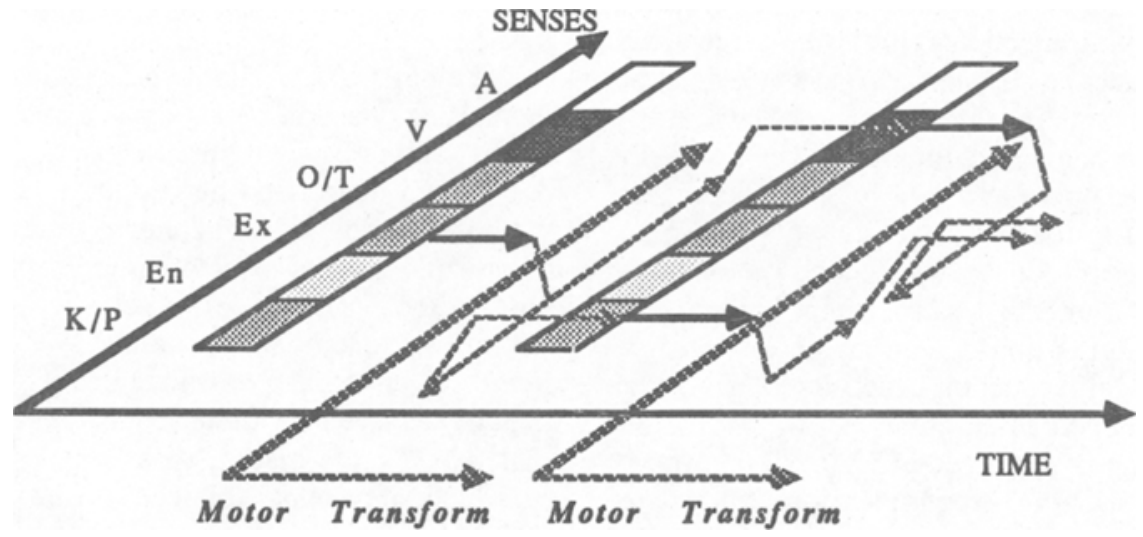

Figure 6. Concatenated motor transform. The prerequisite to goal-directed behavior is the moderation of individual motor-behavior elements and the capacity for temporal concatenation of a sequence of percept-action 'cycles.'

in the bodily repertoire available for use. One only has to observe the flexibility, grace, and fluidity of a child's movement patterns (in comparison to anyone of middle age) to be convinced that the dissociative learning of the motor domain indeed provides an unintended diminution of available performance.

Fortunately, any identified habit can, at least in principle, be readily deconstructed, and dormant elements can be returned to available status, enabling their reconscription to participation in movement generation again. A range of movement re-education programs, arising mainly from rehabilitation work, have become available to interrupt this typical degradative path and return a broad range of motor units to availability. More generally, just being aware of the process, and consciously intervening through pseudorandom and deliberately novel motor actuations at regular intervals, can mitigate the degradative habit of stereotypic body use (see Burton \& Edwards, in press; Mixon \& Burton, 1989), although without a con- siderable training discipline the ballerina's elegant fluidity remains elusive.

\section{Class 4: Skilled Behavior}

What, in essence, is skilled behavior? The essence of skilled behavior is the fluid concatenation of two or more percept-action cycles; that is, the 'enduring' sequentially repeatable association of two or more action elements.

The prerequisite to fluidity in the transition from one action element to the next is the capacity to attend selectively to particular perceptual outcomes of $\left\langle\operatorname{action}_{1}\right\rangle$ to elicit appropriate cues for the initiation of $\left\langle\operatorname{action}_{2}\right\rangle$. General perceptual awareness needs to be inhibited during the exhibition of skilled behavior; otherwise, it would be interrupted. Thus, Class 4 behavior builds upon the coordination and relative temporal phasing of Class 2 behavioral action elements and the selective action repertoire developed through Class 3 behavior, but adds to these the ability to microprogram concatenated motor

Class 4: Skilled behaviour

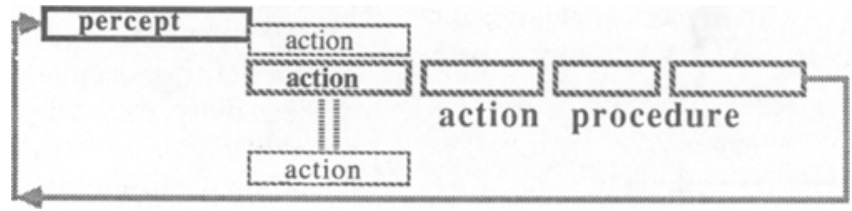

Correlate: Response repertoire, selection, and inhibition of intermediate perception

Figure 7. Class 4 behavior (skilled behavior). Skilled behavior in the form of purposefully monitored action sequences requires the selective activation of a particular motor activity as well as the active inhibition of non-specific-to-the-task perception, which might otherwise activate inappropriate motor transforms. Thus, the prior development of a response repertoire that may be selectively linked to specific perceptually registered features, together with the inhibition of distracting perceptions, affords the possibility of concatenation of motor transform elements, which is the hallmark of skilled behavior (Schultz, 1939/1969). 
transforms that run fluidly from one to the other, by means of a device of inhibiting attention to irrelevant perceptions.

Figure 6 schematizes an elementary concatenated motortransform sequence, building upon Figure 2. The first segment of Figure 6 (in common with Figure 2) portrays the bringing of a touched object into view, creating both visual and kinaesthetic/proprioceptive sensitivity via a motor transform prompted by touch. The second motor transform of Figure 6 is intended to represent bringing the other hand to grasp the object. An example of this sequence is a small child touching, then looking at, then grasping an object such as an apple.

Class 4 behavior is symbolized in Figure 7 in terms of selection from an available response repertoire prompted by a particular context. Instead of cycling back to global perceptual awareness, however, the learner selectively focuses attention on the perceptual outcome of the initial action, which will in turn prime and phase the onset of a second action. Exhibition of such a behavior means a nontrivial specialization of Class 3 behavior. In fact, the primitive selective-action/selective-perception/selectiveaction triplet of Class 4 behavior requires enough experience of the behavior type to be able to 'anticipate' the arising of the derivative perceptual cue from $<$ action $\left._{1}\right\rangle$ in order to prompt the launch of $\left\langle\right.$ action $\left._{2}\right\rangle$ with appropriate timing.

Such behavior is surely the most primitive form of goaldirected behavior. In contrast to the disjointed serial percept and action segments of Figures 3, 4, 5, and 7, goaldirected behavior is symbolized in Figure 8 by the percept and action elements being programmed to overlap. Anticipation is signified by the onset of a perceptual search for a particular (learned by experience) outcome of the initiated action prior to the completion of that action.

Here we begin to recognize for the first time the need for the much-vaunted 'programming and planning' capacities of the prefrontal lobes (Levine, 1986; Milner, 1982; Müller, 1985; Pribram, 1987). Accordingly, without being too specific, we add to the scheme of underlying and subconscious sensorimotor learning of Classes 1-4 the possibility of a logical, physiological, and cognitive 'alternative path' to indirectly mediate in the anticipation and later planning that is necessary for higher behavioral competence. Here, of course, we come back to touch on the

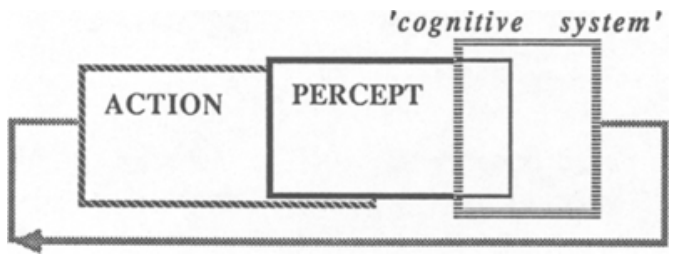

\section{Correlate: Goal-directed system}

Figure 8. 'Overlapped' percept and action. The independent control of the sensory preprocessing system and the motor transform system gives rise to the possibility of the two systems acting in synchrony by means of a coordinated interplay of percept and action sequences. Such activity is what underlies goal-directed behavior. critical dual role of language that was discussed in the first paper.

The elementary goal-directedness involved in selective monitoring of action for anticipated outcomes (anticipated, that is, on the basis of past experience) is symbolized in overlapped action and perception in Figure 8, whereas the possible role of higher level frontal cortex involvement in planning or some other indirect mediation (Luria, $1961,1966,1973,1976)$, is schematized in terms of the 'cognitive system' functionality. We are naturally very interested in defining this functionality more closely, particularly as it relates to the acquisition of skills. We know that many skills mature to a manner representable by 'production systems' (Newell \& Simon, 1972; Newell, 1973), but we are interested here mainly in the earliest phases of skill development which are precursor to such matured and concisely representable skill.

\section{Objectification of Skills}

Although skills are ultimately embodied, their acquisition and refinement usually require orchestration through a tutor or self-tuition. The act of tutoring, of instructing, serves to direct attention, to focus attention on the element of activity to be adjusted. Success in the endeavor is dependent, operationally, upon the initial capacity to follow the tutor's instructions (Hewes, 1973; Noble, 1987), demonstrations, or guidance.

In general, motor-skill development proceeds by means of a tutor's modeling and guided instruction, and then by a more personal process of progressive refinement-with increasing automation of the abstracted action 'prototype'-of the movement components that are working. Correction may require a deconstruction of the movement procedure to enable the learner to refocus selectively from one errant component of the whole movement, the construction of a modification, and subsequent reunification of the corrected component into the whole.

Many activities require that proceduralization occur over a sequence of separate movements. More generally, this process has to operate over a parallel coordination of independent movements.

In the case of both an individual motor skill and a more complex activity sequence, the conscious focus on the movement, if it occurs at all, seems to relate to the onset of the activity, rather than to its continuation, at least after the skill has been learned and automated. In fact, it is much more difficult to 'home in' consciously on an intermediate segment of such a movement than on its beginning phases.

In view of this, I believe that it is reasonable to consider the initial phase of any learned action or proceduralized activity as a Type 1 proprioceptive/kinaesthetic object, even though the action or activity as a whole may extend in time over multiple episodes. These Type 1 'objects of action' seem to me to be rather similar in formal character to our more familiar Type 1 object intensions representing ordinary concrete objects (things), or our concept intensions (ideas). Thus, the initiation of a Type 1 
object of action would seem only to require the learner to focus on its beginning, whereupon the action or activity would ensue and proceed smoothly to completion without the need for further conscious intervention, had it already become automated. For a partially proceduralized but not yet automated movement sequence, in order to remain on track, (conscious?) attention would be required at the end of each movement interval to select and time the initiation of the subsequent movement of the sequence. But the intermediate-attention requirement will presumably fade out as the procedure becomes automated through repetitive use.

Even though they are internally generated, these objects of action will, like their conceptual or objective counterparts, be intensional and will be characterized by a range of perceptual attributes and associations that can be learned through experience of these activated objects. Thus, although they may begin by having a dissociated limitation in the proprioceptive/kinaesthetic modalities, these objects can come to acquire associated attributes from other modalities in a natural way, once conceptual learning is enabled.

If this analysis is acceptable, then there seems to be no barrier to considering all of the object intensions with which the brain is concerned in an equivalent way. That is, physical object intensions, conceptual object intensions, and action object intensions become, in a real sense, isomorphic as the units of currency of the brain's operation.

\section{Class 5: Concentrated Behavior}

Whereas skilled behavior relies upon the inhibition of general perception in favor of focused and selective attention to cues for further action generation, the complementary behavior, which involves inhibition of action in its entirety in favor of concatenated segments of perceptual attention, is exhibited whenever we concentrate to study particular parts of the perceptual environment, as in reading.

Concentrated behavior intervenes in the normal perceptaction cycle to inhibit general action in favor of continuation of particular perceptual monitoring. This is symbolized in Figure 9. It is complementary to skilled behavior in its inhibition of the action, rather than the percept, part

\section{Class 5: Concentration}

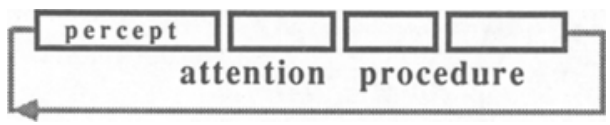

\section{Correlate: inhibition of intermediate action}

Figure 9. Class 5 behavior (concentration). The selective or global inhibition of motor activity between episodes is logically symmetric with the selective inhibition of attention to perceptual elements in Class 4 behavior. Thus, a selective program of perception across episodes can arise, which has the hallmarks of behavior referred to as 'concentration,' as in reading or listening. of the cycle, but it is of course a special case of what in everyday terms would be called skilled behavior. The ability to concentrate is a skill that most, but not all, readily learn; the counterexample is the disorders of attention (Geschwind, 1982). Concentration requires, as do all the higher behaviors, the logical and realizable capacity to dissociate from the full polymodality of the perceptual stream down to a subset of perceptual information normally ranging over few perceptual submodalities. ${ }^{11}$

Concentration enables perceptual programs to follow written or spoken material, as in reading or listening over extended intervals. In a sense, skilled behavior in the kinaesthetic/proprioceptive domain could be called concentrated behavior, but the distinction really lies in the extent of anticipation possible. Thus, experience and individual control is rather higher in self-monitoring of one's own motor performance than is the case in listening or reading, where in general one expects to be continually surprised by someone else's productions.

An extremely interesting and important case where Class 4 and Class 5 behaviors are mixed is in the monitoring of one's own utterance or writing, where a high level of surprise is also rather common. We do not know what we will say until we have heard what we say. And it is impossible to have fluid speech in a conceptual domain without a lot of practice; reading is not enough, except perhaps when rote learning is sought. However, this anticipates a general discussion of conceptual and symboldriven behaviors, and thus we return to our early behavior taxonomy.

\section{Class 6: Deliberate Behavior}

Deliberate behavior is characterized by choice across alternative salient perceptions, not just the more mechanistic-or impressionistic-selection of any single perception as the prompt for action. In deliberate behavior, components representative of earlier behavior classes can be involved, particularly those of Classes 4 and 5. This affords both concatenation over, and coordination of, lower behavior elements to yield extended Type 2 and polyarticulated Type 1 behavior compounds.

The choice in the action domain that has first become available in Class 5 as perceptual concentration through inhibition of action now emerges as an important element of the available action repertoire-that is, inaction.

At this stage, the learner is clearly competent to manage a chosen trajectory through the perceptual environment, and most observable behavior of children up to 2 years of age can be classified into one of the categories included in Figure 10 if we simply agree that the respective sets $\left\{<\right.$ percept $\left.\left._{i}\right\rangle\right\}$ and $\left\{\left\langle\right.\right.$ action $\left.\left._{j}\right\rangle\right\}$ may include attention procedures or action procedures as well as elementary percepts or actions.

The inclusion of attention procedures or skillful activities (Classes 5 and 4, respectively) in separate behavioral classes that may be subsumed in Class 6 is supported by recent data from Nissen, Knopman, and Schacter (1987), who illustrated the neurochemical dissociation 


\section{Class 6: Deliberate behaviour}

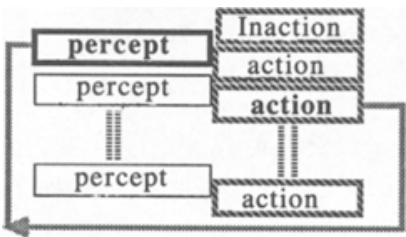

\section{Correlate: Selective attention; choice of response repertoire}

Figure 10. Class 6 behavior (deliberate behavior). Class 6 behavior is a compound of Class 4 and Class 5 behaviors, characterized by selectively programmed inhibition of inappropriate motor activity and programmed attention to avoid distracting perceptions. Deliberate behavior comprises a search for specific goal perceptions.

of 'procedural' memory from object-related 'declarative' memory. ${ }^{12}$

The capacity for concentration on a limited range of perceptual information from the environment undoubtedly enhances the rate of sensorimotor learning and presumably underlies the efficient utilization of neurones of the temporal association cortex. ${ }^{13}$

\section{Proceduralization}

The capacity for concentrated attention in combination with more general action procedures underlies the capacity for proceduralization of elementary concatenated action elements. Figure 11 is a schematic representation of an extended percept-action sequence that might be observed in the course of early learning. With each successive episode of a play 'routine' within a domain there lies the possibility of one new articulated perceptual motor attribute relating to an object in the perceptual environment, by means of the normal perceptual learning process of dissociation. The labels of Figure 11 intervening between each episode are of two forms; those in capitals (e.g., 'PLAY') signify views formed of 'significant' behaviors by an external observer, and those in lower case (e.g., 'existence,' where the object is first noticed) correspond to internally articulated attributes of the Type 1 perceptual object discerned from the perception sequence impinging on several modalities.

As previously discussed (in the first paper) in the case of auditory Type 2 objects, where rehearing of the agent's utterance is necessary for any articulation of temporal attributes to the Type 2 object, at least one replay of the percept-action sequence of Figure 11 is necessary (though possibly not sufficient) for articulation of known temporal substructure to the procedure. Figure 12 illustrates the role of a replay of the sequence with slightly changed global context in providing for the articulation of (at most) one temporal substructure attribute (of the many possible) of this Type 2 object. Although observers may be aware of formal structure within the sequence, this cannot become known to the learner personally in the absence of at least one replay. ${ }^{14}$ In order for structure to become known within an observably coherent play segment, a replay of some or all of the sequence delineated by the typical disjunctive switches of attention in play between different objects is required. Figure 12 thus shows

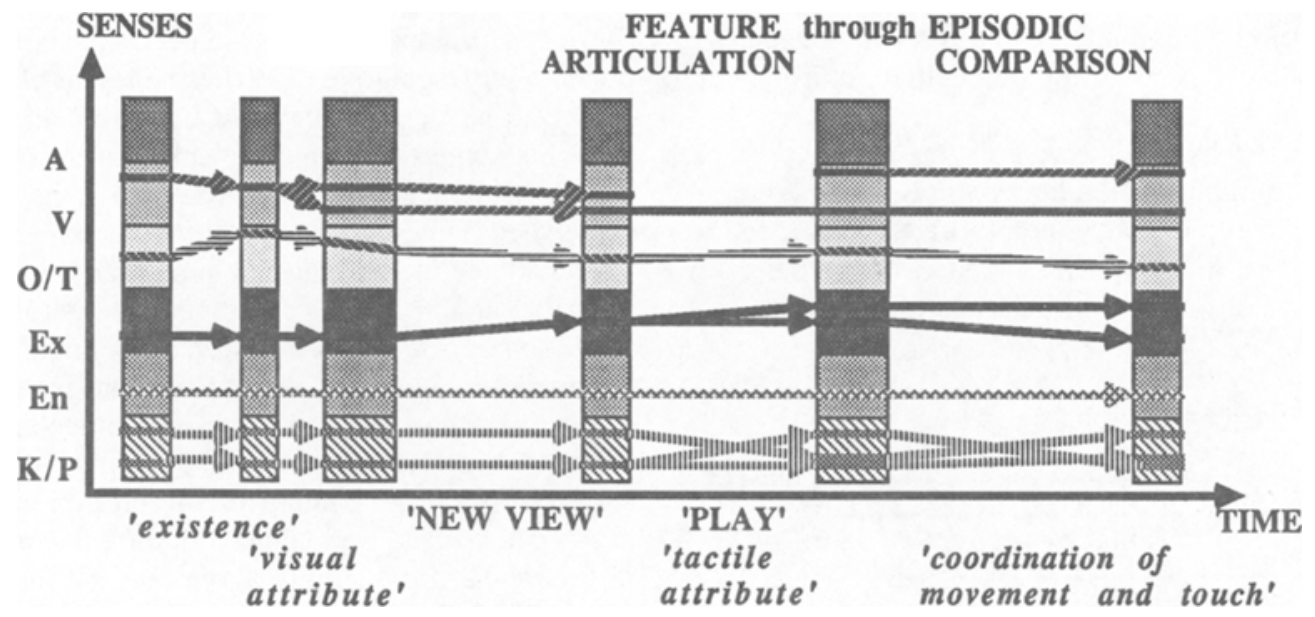

Figure 11. A percept-action sequence. This diagram represents a synthesis of the concept of growth in intensionality for a perceptual object (see preceding paper, Figure 4) and the concept of active learning implicitly associated with the perceptual transformation role of the motor transform of Figure 2 of the present paper. That is, the conscious or unconsciously generated action comprising the (selective) motor transform is grafted onto the basic computational logic of perceptual knowledge acquisition, in the form of a cyclic interplay of percept and action. Here, the perceptual learner is no longer cast in the passive receptor role; we can explicitly address real-time activity sequences that comprise coherent behavior in a given domain. 


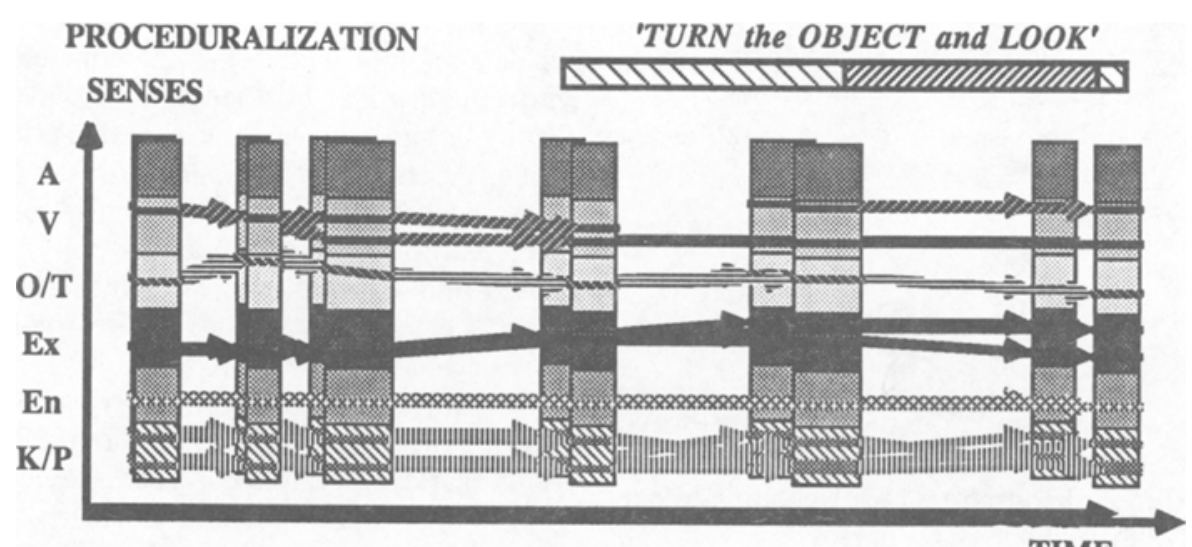

TIME

Figure 12. Proceduralization. Here one sees again the consistent logical limitation imposed by the episode-comparison logic: that only one 'chunk' of information becomes articulable from each successive, mutually relevant extended experience segment. The capacity to objectify successfully through progressive temporal feature articulation, eventually (but not immediately) demonstrable by the capacity to reactivate the percept-action sequence selectively, must be acknowledged to be the prerequisite to any purposeful proceduralization of action sequences into activities.

the temporal segmentation of the percept-action concatenation from the undifferentiated sequence to the first known temporal articulation. The first occurrence is just stored data. It becomes 'objectified' through recognition (and change) in the context of a recurrence, which can lead to the articulation of at most one temporal feature with respect to the otherwise unified trace of the initial data. This might be denoted by an observer as 'TURN the OBJECT and LOOK' as is indicated above the Type 2 object at the top of Figure 12. ${ }^{15}$

The learner's analysis of novel percept-action sequences, which begin as basically random sequences, cannot reasonably be achieved on a single pass; this would require the capacity to store articulated objectification of all such sequences, many never to be meaningful or useful. The recurrence requirement provides the necessary noise-rejection filter on what is objectified in memory as a Type 2 object intension. This is isomorphic with the noise-rejection logic operating through episodic gating and recurrences of Type 1 objects, which reminds us that recurrence with (at least some degree of) novelty with respect to a previously encoded trace is explicitly required to provide a basis for temporal articulation of the internally constructed representation of the percept-action sequence, the activity object intension.

It may seem curious for this point to be labored when the sequence has, in its schematic form, obvious temporal structure. Although structure may be apparent to an observer, no such structure will be apparent automatically to the learner, and in particular the actual episodic basis of brain processing itself will be completely imperceptible to the learner. The learner has no need for (though possibly much interest in) warning or noting of actual episodic gating. The mechanism of operation can, if it works, be entirely hidden. For this reason, I have continued to insist that the temporal gating of the perceptual process- ing system operates on a subconscious basis; it has certainly not been obvious, despite its logical necessity.

\section{EARLY MOVEMENT QUANTITATION AND CONTROL}

The submodalities of the kinaesthetic/proprioceptive $(\mathrm{K} / \mathrm{P})$ sense can in principle be resolved down to the 'motor unit' (Rothwell, 1987, p. 31), which symbolizes the smallest unit of muscular contraction. This is the smallest number of fibers that can be activated by the central nervous system (CNS) at any one time. In mammalian skeletal muscle, the primary organization of the muscle is imposed by the CNS, and each motor unit comprises a single motoneurone axon, its terminal branches, and the associated muscle fibers.

The actions of the behavior taxonomy can all be considered to be based on $\mathrm{K} / \mathrm{P}$ objects, which span the entire domain of motor units. These Type $1 \mathrm{~K} / \mathrm{P}$ (or proprioceptive) objects differ in the motor units that are actuated in the corresponding action, and thus correspond to different types of motion that can be initiated in a given motor transform. ${ }^{16}$

The temporal articulation and extent ${ }^{17}$ of any 'action'as a Type $2 \mathrm{~K} / \mathrm{P}$ (or kinaesthetic) object-is described in terms of the 'movement unit,' examples of which have been described for human infants by von Hofsten (1979) and by Fetters and Todd (1987). The kinaesthetic objects corresponding to selected movements such as reaching are observed in execution to be articulated into movement units.

For the 5-9-month-olds studied by Fetters and Todd (1987), both the average number of movement units per reach to a static target-increased concatenation-and the mean duration per reach increased to some extent, as shown in Table 1. Reaching movements in 5-9-month- 
Table 1

Infant Reaching Movements (Static Target)

\begin{tabular}{cccccc}
\hline & \multicolumn{2}{c}{ Near Reach } & & \multicolumn{2}{c}{ Far Reach } \\
\cline { 2 - 3 } \cline { 5 - 6 } Age & $\begin{array}{c}\text { Duration } \\
\text { (in msec) }\end{array}$ & $\begin{array}{c}\text { Movement } \\
\text { Units }\end{array}$ & & $\begin{array}{c}\text { Duration } \\
\text { (in msec) }\end{array}$ & $\begin{array}{c}\text { Movement } \\
\text { Units }\end{array}$ \\
\hline 5 months & 548 & 2.1 & & 714 & 2.2 \\
7 months & 779 & 3.2 & & 777 & 2.4 \\
9 months & 769 & 2.8 & & 868 & 3.4 \\
\hline
\end{tabular}

Note-Data are from Fetters and Todd (1987).

old infants show a tight coupling in the curvature-speed relationship. Individual movement units are tightly clustered around $200 \mathrm{msec}$ in duration, corresponding to one acceleration and one deceleration (Fellers \& Todd, 1987).

Among the younger infants studied by von Hofsten (1979) with moving stimuli, variation from the individual reaches of 15-21-week-old infants to those of the older infants around 9 months of age provides evidence of a dissociative learning capacity in Type 2 kinaesthetic objects of movement in reducing the number of movement units per reach (from 3-5 to 1-2), and in the movement units themselves, from the earlier more ballistic to the later more refined movements. Individual movement-unit durations were determined to be relatively stable in the $350-400-m s e c$ range for the younger infants. However, the later temporal pattern in movement indicated that a phasic refinement process had occurred in which the initial movement unit of a reach in the older infants was considerably longer than subsequent movement units. In contrast to the undifferentiated movement-unit durations in the younger infants, the older infants exhibited reaches initiated by a movement unit of some $500 \mathrm{msec}$, whereas subsequent movement units were only $250 \mathrm{msec}$ long.

We will take these human infant reach durations as indicative of the time scale of motor transforms in general, and will ascribe the duration of elementary actions of the behavior taxonomy presented here to be on the order of 0.5-0.9 sec. This means that elementary actions are rather time consuming on the electrophysiologist's time scale.

With regard to the sensing of movement, according to Rothwell (1987), the three classes of converging afferent input to the spinal reflex pathways exhibit presynaptic inhibition of all of the afferent fibers.

These actions are long lasting (up to $1 \mathrm{sec}$ ), widespread and produced by polysynaptic spinal pathways which have yet to be identified. As one might expect, the interneurones of these pathways show convergence from many sources. A precise function for presynaptic inhibition in motor control has yet to be established. (Rothwell, 1987, p. 124)

We will take this as evidence supporting phasic perceptaction cycling as required by our behavior taxonomy.

\section{SPECIFIC NEUROTRANSMITTER AND NEUROANATOMICAL FUNCTIONALITY}

The cerebellum seems to be clearly implicated in the evident capacity of the infant to refine and 'fuse'18 elementary movement units into a continuous action, to phasically program successive movement units so that they concatenate smoothly, and to modulate the extent of particular movement units. Furthermore, the availability of fluid action comprising several movement units requires continued cerebellar participation. For example, ataxia of the arms can be manifest when normally smooth movements, such as moving out to touch an object, become decomposed into several steps (Rothwell, 1987, p. 273). With cerebellar dysfunction, we see reversion to the infant's early staccato movement style.

Presumably, cerebellar circuitry available to refine individual actions determines the range of fluid coordinations that are possible for each species. ${ }^{19}$

Although the cerebellum is clearly implicated in the phasing and timing of individual movement units within an action, given the selected extension of the initial movement unit in an action as shown by von Hofsten (1979), we must also look to the cerebellum for evidence of gating corresponding to the initiation of each action. ${ }^{20}$

The obvious candidate elements for this action gating are the climbing fibers. According to Rothwell (1987, p. 262):

During many types of active movement, there is little modulation in the firing rate of climbing fibres. Complex spikes appear in the Purkinje cells at a steady slow rate of 0.5 to $2 \mathrm{~Hz}$. Despite the low level of activity in the climbing fibre system, the climbing fibres are essential to the proper operation of the cerebellum. Removal of the inferior olive, with subsequent degeneration of the climbing fibres produces symptoms in experimental animals that are indistinguishable from removal of the cerebellum itself. Climbing fibre input, although infrequent, and with little immediate effect on Purkinje cell discharge rates, must have some vital long term influence on cerebellar function. (p. 262)

Cerebellar function is implicated in movement timing, learning, and coordination (Llinas, 1981). Our interpretation of cerebellar function is that the motor object-the Type 1 proprioceptive object-which is to be activated to effect each action has motor-unit elements whose durations are controlled by their own Purkinje cells. Local temporal concatenation of movement units and the fusion of movement units into a fluid unitary action-the Type 2 kinaesthetic character of each action-is the role of cerebellar function, but the phasic timing of onset of the unitized action is determined by the climbing fibers from the inferior olive. ${ }^{21}$

In formal terms, the Type 1 object broadcast from the motor cortex and the basal ganglia determines the action type. The Type 2 objective character-or 'execution plan'-of the movement itself is determined by the dynamics of intracerebellar connections. When the movement action is initiated is determined by the climbing fibers.

Our previous discussion of overlapped, rather than serial, percept and action components from elementary behavior classes (see Figure 8) concerned the necessity of monitoring the outcomes of each activated action element for appropriate cues in order to time the launch of the subsequent action element of an extended activity. We can therefore now attribute exactly the same logical re- 
quirement to the programming of movement units within an action, but the link from one movement unit to the next in this case has no requirement for a general sensory monitoring via the global perceptual apparatus. The cerebellum merely specializes the general model to the case of smoothly programmed fine movement (involving few movement units) within an action. Conversely, in purely conceptual terms, we see the onset of skilled Class 4 behavior, which comprises concatenation of action elements as a generalization of the cerebellar capacity for intraaction movement unit to movement unit programming, to the inter-action case. Inter-action (action followed by action) coordination, structured into the classes of our taxonomy, is the brain's whole purpose.

Such a capacity requires that the action objects (Type 1) that define the actions be linked in order to be independently selectable (Class 3 behavior) in the appropriate sequence. The basis of any such selection has to be prior dissociation of some elements from a coordinated motor object (Class 2 behavior) through Type 1 learning. Most of the evidence for programming of sequences points to the involvement of the frontal cortices.

The direct origin of the 'broadcast' of the motor object to the cerebellum is the basal ganglia; the activity of motoneurones in the basal ganglia determines the content of the Type 1 object to be initiated and controlled by the cerebellum.

In summary, many neurones in the basal ganglia are phasically active in relation to movement of specific body parts. Because of their specificity, these neurones cannot be providing any tonic or modulating influence over some general aspect of movement control. However, since their firing rate is only rarely directly related to the pattern of EMG activity in specific muscles, they are unlikely to provide any direct input to control muscle activity. (Rothwell, 1987, p. 301)

The motoneurones of the basal ganglia must serve to determine the identity of motor units that will comprise an action. In this role, their firing rate need have no relation to the actual EMG activity of the muscles to which they refer. The relevant Purkinje cells of the cerebellum are where that correlation, or rather control, should arise.

The phasic activation of an action in response to a particular percept is accomplished in the present model by two independent elements. First, definition of the action object is determined by the basal ganglia (in communication with the motor cortex), and second, a global gating signal for initiation of the action is required. The gating for the action initiation is proposed to involve cerebellar climbing fibers, whereas actual movement control (phasic initiation, duration control, and smooth transition between movement units, together with any required coordination and coarticulation) is effected within the cerebellum to ensure that (locally) smooth and fluid action will arise from the activated concatenations of movement units.

The definition of the action object for each basic percept-action cycle is conveyed by the output nuclei of the basal ganglia (the globus pallidus and the substantia nigra). The attribution of an action-definition role (rather than a motor-control role) to the pallidal and nigral outputs is supported by the lack of induced tremor or tonic muscle changes with either chemical or electrolytic lesions of these output nuclei (Rothwell, 1987, p. 306).

In the psychonomic theory, each defined action object arises phasically in relation to a globally gated episodic percept. The theory relies upon essentially arbitrary linkability of motor unit to perceptual submodality, and the 'switchboard' that achieves any specific link of a perceptual submodality to a particular motor unit must be in the input units of the basal ganglia-that is, in the caudate nucleus, the putamen, the hypothalamic nucleus, and the ventral striatum (Heimer, 1978).

The putamen receives input from the sensorimotor areas of the cortex, whereas the caudate receives input from the frontal and association areas of the cortex. ${ }^{22}$ There is a clear topographical demarcation of cortical inputs at the level of the caudate and putamen, exemplified by the inferotemporal areas projecting to the tail of the caudate, the sensorimotor and premotor areas projecting to the putamen, and the prefrontal areas projecting to the head of the caudate. These two nuclei are the phylogenetically newer parts of the basal ganglia, and are jointly named the neostriatum (or simply, striatum).

The inputs to the basal ganglia from the cortex do not control movement, because they mostly tend ${ }^{23}$ to fire after the onset ${ }^{24}$ of movement as monitored by EMG. ${ }^{25}$ This supports the action-definition notion of the basal ganglia function. The temporally overlapped action-percept structure of the goal-directed scheme illustrated in Figure 8 above is the way of reconciling the delayed involvement of the motor cells of the basal ganglia (in the presumably familiar movements under test): the end of an active action is monitored in order to serve as a sensory cue for selective activation of a subsequent action.

The 'context-dependent cells' of the basal ganglia draw their inputs polymodally:

The discharge pattern in the remainder of the neurones in globus pallidus, putamen, substantia nigra pars reticulata and subthalamic nucleus is not influenced by movement alone. In this respect they resemble the majority of neurones in the caudate and substantia nigra pars compacta. It is now believed that some of these neurones respond to behaviourally significant stimuli, rather than to movement per se. There are neurones within the caudate nucleus with complex properties which correspond to more than one modality of sensory stimulation. (Rothwell, 1987, p. 298)

Many of the movement-related cells of the putamen, globus pallidus, and subthalamic nucleus also respond specifically to somatosensory stimuli.

The fact that most cells with sensory input also respond to movement implies a convergence of sensory and motor input from different cortical areas. However, there are a small number of cells which respond either to somatosensory input or to movement. There is then incomplete convergence within the basal ganglia. (Rothwell, 1987, p. 298)

The thalamocortical/corticostriatal projections are those that provide input for selective action definition. We 
then have in the striatum the 'switchboard' that interconnects, ${ }^{26}$ within the dynamics of a single perceptaction cycle, an 'input' polymodal percept to an 'output' action object. The densely interconnected striatal neurones-which show recently quantified input and output synaptic densities of $30,000-40,000$ per neuroneeffect the percept-action switchboard, but this connection must be qualified by reference to cortically stored information, which is activated by the primary thalamocortical projection.

The general polymodal concept of 'percept' in the psychonomic framework requires input from all six senses to be available for the selective formation of percept-action objects; all that is required is the inclusion of the olfactory 'ventral striatum' and the subthalamic nuclei (mediating the endoceptive submodalities) in our general definition of the striatum.

The second input route to the basal ganglia is from the thalamus. In the first paper, I pointed to the likely significance of the thalamostriatal focal aggregations (Jones, 1985 , p. 116) in episodic gating of the whole perceptual system.

The episodic gating of the first paper was discussed in the restricted terms of the cortical memory formation of episode-based objects, which, in principle, cover all six senses. With the current extended framework being more focused on behavior generated selectively from percepts, rather than on percepts themselves, we need to broaden and extend the discussion of the episodic gating model.

Episodic gating affords clear relative quantification of sensory inputs via perceptual information channels and, via a two-neurotransmitter involvement, encodes episodes cortically through combination of thalamocortical primary projection to sensitize the cortically specific terminal for each channel and a second 'proceed-to-store' signal, which is broadcast (via noradrenaline from the locus coeruleus) globally across the cortex to capture the transient excita- tion wherever primary projection may have occurred within the episode.

It is now useful to broaden the gating hypothesis from global storage initiation to include concomitant transient inhibition of the sensory acquisition system. The reason for this is that if any action, which is 'launched' on the basis of that sensory episode through a percept-action link, is to be monitored, then effective monitoring of the direct effects of the associated motor transform would be most clearly definable against a minimal background of irrelevant inflow to the CNS. In a similar vein, the specificity of any action actually launched in response to a percept will be heightened by concomitant inhibition of prior motor activity as the new action was launched.

The extent to which such ideal 'background minimization' through central inhibition is actually realized in the sensory or the motor domains in the working brain is bound to be difficult to assess whenever, through learning, extensive coordination of independent activities is exhibited. However, during early learning, when the explicit programming of primary percept-action links is being achieved, one might expect such phasic behavior in either domain to be more apparent. One notes in particular the regularity of the human EEG $1-2-\mathrm{Hz}$ delta wave in infancy and again in 'senility' (e.g., Alzheimer's disease), or under anaesthesia. The unrecognizability of this EEG component in waking in the intervening years is of course a major puzzle. ${ }^{27}$

At the earliest stages of learning, the simplest logic would involve unitary gating of the sensory system, with a centrally mediated quietude being imposed on the sensory apparatus for a short period. ${ }^{28}$ Exactly the same gating signal could trigger phasic activation of new motor elements.

To complete the logic, launched actions should equally be suppressed at some later stage in order to return focus to new perception against a quiet background. However,

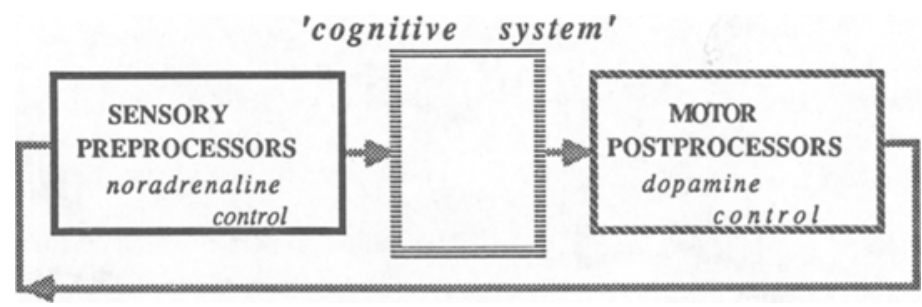

Figure 13. Independent percept and action control. The distinction between Class 4 and Class 5 behavior and the richness of the possibilities of Class 6 behavior depend on the possibility of independent inhibition of perceptual processing and of motor processing. Thus, we can generalize from the concept of sensory preprocessors and motor postprocessors supporting the episode-processing logic to the active moderation of the involvement of either factor in a given perceptaction cycle. This moderation could only be achieved by globally active neurotransmitter networks. The noradrenaline network is proposed as the moderator of perception, and the dopamine network is proposed as the moderator of the motor system. The serotonin network presumably tunes the activity level of both of these independent networks throughout the sleep/wake cycle. (See McRae-Degueurce, Dennis, Leger, \& Scatton, 1985, on the regulation of rat locus coeruleus activity by serotonergic activity.) 
this seems achievable (given the data of Fetters \& Todd, 1987) by the simpler expedient of launching actions as delimited sequences of movement units that do not typically extend beyond the duration of the normal gating cycle.

Such simple and unitary gating would apply to the temporally minimal percept-action cycles of behavior in Classes 1-3. For the higher behaviors, independent control of both the perceptual acquisition (noradrenaline) and the motor system (dopamine) is required. This is illustrated schematically in Figure 13. (The dopamine system is not as unitary as the noradrenaline system, showing five subsystems with differing roles.) The generally inhibitory roles of both noradrenaline and dopamine fit with this general gating hypothesis.

Although greater inhibition of action by dopamine might seem contrary to the aim of more effective action control, this simply allows for more gating, more independent episodes, and thus more opportunity to initiate independently controllable actions.

Interestingly, the biogenic origins of noradrenaline from dopamine (via dopamine $\beta$-hydroxylase), which is in reverse order if percept invariably precedes action, is quite readily understood again with the aid of Figure 8 . The launch of a broadly based action object requires the release of large amounts of dopamine for its control. Such a large action will have the result of considerable perceptual change, so that the perceptual apparatus will be activated in a widespread manner. To bring this activity back to quietude requires a widespread availability of noradrenaline: Marona-Lewicka, Michaluk, Antkiewicz-Michaluk, and Vetaluni (1987) have demonstrated the relationship between excitability and noradrenaline (NA) availability, in a comparison of two species of mouse, ${ }^{29}$ which supports this view of NA's functionality.

In the simple gating paradigm, NA rising in the brainstem's locus coeruleus is attributed the central role. The central, but so far diverse and somewhat perplexing role of the locus coeruleus has been documented in many recent studies. ${ }^{30}$ The NA system projects forward through the forebrain before projecting tangentially across the sensory cortex. The integration (perhaps interjection) of planning information from the frontal cortices, together with high-level sensory information from the temporal association areas, is probably achieved by the caudate nucleus projecting onto the small but vital amygdala, which broadcasts via the stria terminalis on to the movement-specific parts of the basal ganglia.

This central anatomical position of the amygdala, together with the profound deficits that arise from its ablation, exemplified by the Bucy-Klüver Syndrome ${ }^{31}$ associated with bilateral temporal lobectomy, leads us to attribute to it the central role of coordination in the temporal domain. The broadcast of the global NA gating and proceed-to-store signal arises in the locus coeruleus; however, in this model it is triggered by the amygdala. ${ }^{32}$ This leads to the conclusion that the generalized thalamostriatal take-outs (the focal thalamostriatal aggregations of Jones, 1985) are prime candidates for the role of 'informing' the amygdala of appropriate times (with respect to minimal novelty and multiple independent channels as per the requirements of the previous paper) for the global epısodic gating signal to be broadcast, as we have already discussed (see previous paper).

The psychonomic theory suggests an important distinction between the temporal structure of the primary sensory projections to the cortex and derived corticostriatal communication, and the temporal structure of the global proceed-to-store signal. To employ a digital computing metaphor, one can distinguish the basic cycle time of the 'machine' from the instruction time-which may be many 'clock cycles.' In any one of the independent sensory-input channels, a sequence of 'spikes' may be required to reach the point where an 'episodic trace' is recognized and the proceed-to-store signal is issued. Using this metaphor, I would propose to associate acetylcholine with the clock cycle of the episodic processor, while associating noradrenaline with the 'instruction cycle,' gating episodic storage initiation. The episodic gating signal via such a divergent neurotransmitter system would have the following significance: Enough salient information is present to define a novel episode-proceed to store wherever perceptual information has been registered. ${ }^{33}$

Neurochemically specific and temporally resolved intraepisodic experiments during defined behaviors are required before clarity can emerge in the precise functionality of acetylcholine. The same problem arises in the attribution of detailed functionality of the multiplicity of individual neurotransmitter or neuromodulator molecules that cooperate in the execution of real-time processing in the brain. The densest staining of acetylcholine markers occurs in the striatum. In the psychonomic model, acetylcholine is therefore assigned the basic signaling role of intraepisode processing at the midbrain level. Acetylcholine must operate without confusion from the global gating signal propagation and without confusion from the primary thalamocortical signal propagation. Its unique chemical properties are quite dissimilar to those of the dopamine, noradrenaline, and serotonin triad, which are chemical and biochemical specializations of a particular basic structure. Figure 14 compares noradrenaline and acetylcholine structures; dopamine differs from noradrenaline just by the lack of the sidechain hydroxy substituent, whereas serotonin (5-hydroxytryptamine) lacks a ring- $\mathrm{OH}$ and has a cyclized, oxidized, and differently substituted sidechain at what would be noradrenaline's hydroxy-substituted sidechain carbon.

At the present level of detail, it is not yet possible to favor one of the several possible explicit functional roles available in the model for acetylcholine (e.g., acetylcholine activity could drive one- or two-way striatal-cortical direct communication, or it could simply respond to corticostriatal signaling prompted by the primary thalamocortical projection). In any case, the high-frequency activity of acetylcholine in this model would be closely associated with the 8-13-Hz EEG alpha waves.

The psychonomic model provides a clear pointer to noradrenaline's role, even though the basic theory is cast at a sufficiently general level that comment on detailed 

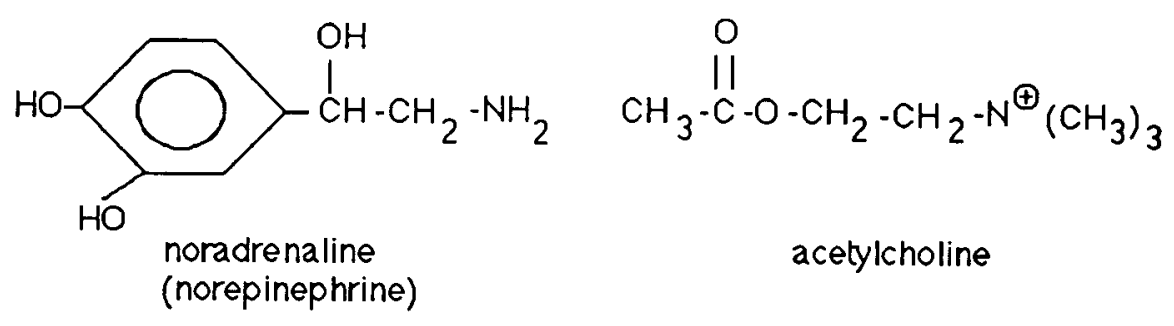

Figure 14. Processor instruction cycle neurotransmitter structure (noradrenaline) versus processor clock cycle neurotransmitter structure (acetylcholine).

functionality of many other transmitters or modulators is not yet possible. Given that experimental dissociation of noradrenaline's and dopamine's roles in coherent brain activity has been difficult, this attribution of explicit functionality to noradrenaline, which makes it the key reference neurotransmitter system from which to measure all coherent intraepisodic activity in the brain, is expected to be of value in future neurochemically, physiologically, behaviorally, and temporally resolved experiments on brain activity.

Since the noradrenaline system rises in the locus coeruleus of the brainstem, the assessment-of-datasignificance signal to gate the episode must involve a 'take off' from the thalamocortical primary projection, in a system connected to the locus coeruleus. The striatum would fulfill this role, as was earlier suggested, with the aid of the polymodal claustrum (Boyapati \& Henry, 1985; Olson \& Graybiel, 1980). As Jones (1985) has noted, "The terminal ramifications of the thalamostriatal fibers are not diffusely distributed. Instead, bundles of fibers end in small focal aggregations separated by gaps." The striatum is given the role of monitoring the 'general quantification' of information across submodalities without toodetailed a regard ${ }^{34}$ to the feature specificity of the perceptually registered data. The polymodality of the claustrum would then be associated with aggregation across modalities: communication with the striatum would, given abovethreshold information across the range of modalities, lead to the noradrenaline episodic gating from the locus coeruleus. One would then associate the $1-3-\mathrm{Hz}$ delta wave of EEG with the noradrenaline signal.

The implementation of an inheritance-based format for episodic memory requires a contingency analysis regarding the relevance of the current episode to previously encoded/stored episodes. Thus, a totally novel context to an episode would lead to 'straight-through' memory encoding of the perceptual data, whereas a new episode in a known domain would be differentially stored by reference to one or more earlier and recalled episodes in that domain. It is appropriate to attribute to a polymodal 'unit' ${ }^{35}$ the role of mutual relevance assessment between currently registered and stored episodes: the achievement of above-threshold relevance would have (1) an affective component through the hypothalamus, involving feed- forward to the frontal cortex, since the generation of activity types should also be contingent upon novelty versus previous relevance of each episode, and (2) a store-byreference component, which quite likely would involve invocation of the hippocampus. The hippocampus would then be conditionally involved in learning, involving only episodes in domains already known, and its stereotyped lamellar architecture would be responsible for the logic of the store by reference (without duplication or confusion) of sequences of mutually relevant episodes back at the cortical level.

Furthermore, one has to attribute to the hippocampus the role required in this overall framework of managing the formation, or selective enhancement, of the explicit percept-action connections within the striatal switchboard required for and at the base of all learning. The EEG theta waves in the intermediate $3-8-\mathrm{Hz}$ range, which are closely associated with the hippocampus and long-term potentiation, are attributed the role of strengthening synaptic connections in the striatum to realize the micromodal links between percept and action elements, which on the basis of past experience and at least one recurrence are "expected' to have future utility.

Finally, one would have the frontal and prefrontal cortex (Levine, 1986; Milner, 1982; Müller, 1985) involved in action selection (and temporal programming of action sequences, leading to proceduralization and automation). The range of choices for selective attention/action must somehow be determined from the 'geometry' of protostorage of the current episode relative to its reference episode, which would in turn depend on the structure of overlap between the two episodic vectors, each having vector components across the submodal features available at the CCS.

In the forebrain, percept-action sequences that have recurred are progressively articulated as activities; this is where our Type 2 proceduralization over actions is planned and organized. In general terms, encoding elaboration of such activities can be achieved by giving priority to selected action whenever it arises in the context of a predefined percept occurring (or expected to recur) within a familiar activity sequence; that is, within a familiar Type 2 object. Such priority would favor Type 2 learning at the level of multi-episode activity. As always, selec- 
tion of activities from a repertoire requires preliminary Type 1 learning. Since all parts of the sensory and motor cortices, the diencephalon, and the lower brain are fully committed to intraepisodic and episodic processing, only the forebrain-in particular the prefrontal cortex-has uncommitted capacity to manage learning and the encoding of Type 1 and Type 2 knowledge relating to multiepisode activities.

The necessary priority is achieved simply by having the noradrenaline network project first over the frontal lobes before going back over the motor and sensory parts of the cortex. Since priority must be given to maintenance of initiated multiepisodic activity over simple perceptaction cycling of a more general nature, the broadcast of the noradrenaline episode-gating signal reaches the forebrain regions of the cortex before it reaches the sensory cortex areas.

In overall temporal structure, the model has the highest frequency activity associated with the brainstem and thalamus sensory-acquisition signal propagation as input, and brainstem and cerebellar signal propagation for the control of motor execution. At this level, we are concerned with processing on the intraepisodic time scale for detailed monitoring and fine control. From the basal ganglia of the midbrain we have the focus of episode processing on an intermediate and slower time scale, on the order of seconds, to mediate the percept-to-action transform that initiates each episode. At the cortical level, we have control of changes at the episodic and multiepisodic time scales, with episode-to-episode programming of experientially organized activity under the control of the prefrontal cortices.

\section{THE EPISODIC TIME SCALE: THE ENDOGENOUS EYEBLINK}

The various categories of object of use in memory have a high degree of isomorphism despite their widely differing sensory modal or temporally modulated content. The brain can generate all of the relevant objects with the same underlying primitive processing mechanism. Typically, in the early stages this processing mechanism must involve explicitly global discrete episodic processing, at least before extended skilled and concentrated activities break up the global unity into specializations and irregularize the temporality of processing; 'global gating' means that some corresponding physiological system for gating must be found to operate globally in the brain. Only recently have explicit neurophysiological studies of sensory gating begun to appear (Adler, Rose, \& Freedman, 1986).

The entire psychonomic theory depends upon this one key ingredient: explicit discretization of the experienced perceptual influx into 'quanta' of variable duration called episodes. In spite of its complete lack of recognition so far, and even if such episodic processing were always wholely subconscious, the critical importance of global gating to the whole system suggests the likelihood of some external manifestation of its existence. But how could such a manifestation have escaped notice, if the psychonomic theory is correct?

The subconscious episodic gating of humans, who are visually dominant, is proposed here to be externally marked by conscription of the eyeblink, in both perceptual learning and conceptual learning. ${ }^{36}$

Armed with our early behavior taxonomy, we can readily predict that the more mature perceptual and conceptual learner, imbued with a wide range of skills in sensory modal processing, would, through familiarity with processing in ever larger 'chunks' and more extended procedures within a domain, blink less frequently than would a neophyte to the same domain ${ }^{37}$ The blink rate is also known to be affected by a range of mental abnormalities. ${ }^{38}$

As Stern, Walrath, and Goldstein (1984) have noted, the endogenous eyeblink (the eyeblink in the absence of an externally identifiable eliciting stimulus) is cortically controlled with respect to rate, form, and temporal distribution in relation to cognitive state variables. ${ }^{39}$ If gating occurs in association with completed acquisition of an episode containing the necessary minimal novelty, and if it is flagged by the eyeblink as is proposed here, any loss of visual information due to the blink itself would be least significant at precisely that time (of episode completion) ${ }^{40}$

But rather than rationalizing its lack of interference with visual perception, we should now look to the endogenous eyeblink as the specific external marker of episodic gating in the segment of the lifecycle whenever conscious cognitive control of episodic gating is apparent (through the absence of a well-defined delta wave in EEG).

In the psychonomic theory of knowledge acquisition, it is the 'data-driven' aspect of episode gating that, requiring a minimal degree of novelty in each new episode which in turn depends on the prior learning of the individual, gives rise to the inevitably variable temporal character to episode processing (and thus to eyeblinks). ${ }^{41}$ That is, it is the intrinsic unpredictability of the eyeblink that has thus far so effectively camouflaged its role as the external signifier of the underlying and fundamental gating of episodes.

Once this particular motor transform has been internally and explicitly discerned (by dissociation from the general perceptual flux due to motor activity), the endogenous eyeblink-like every other motor transform-can be inhibited. ${ }^{42}$ However, this is not usually the case, and eyeblinks occur irregularly as often as every 1-2 sec in active periods, perhaps $10,000-20,000$ times during the waking day.

If global sensory acquisition time-scales are no faster in general than the order of 1-2 sec, this can encompass: 2-3 saccadic eye movements in vision (in effect a preprogrammed 'triangulation' in itself), an inspiration/ expiration cycle or several faster 'sniffs' in olfaction (Freeman, 1981; Freeman \& Schneider, 1982), taste reaction times of 400-850 msec (Kelling \& Halpern, 1987), 
a characteristic affective/emotional time scale of seconds (Clynes, 1977)-and/or the heartbeat rate-in endoception.

Episodes that maintain a general sensitivity across the senses and are relatively quantified in terms of preprocessed perceptually relevant information must await intraepisode quantification of information in each of the senses. The observation of the quiescent period after primary afferent activity in Kauer and Shepherd's (1977) olfaction data (see first paper) and in the long-lasting (1-sec) presynaptic inhibition of motor afferents are both observations consistent with the gating hypothesis we have adopted. The 500-900-msec duration of movement-unit sequences constituting primary motor transforms (actions), the $0.5-2-\mathrm{Hz}$ cerebellar climbing fiber activity, and the similar frequency of activity in the locus coeruleus itself all comply with a sensory gating and the motoreffector time scale of $0.5-2 \mathrm{sec}$ for percept-action cycles.

The disappearance of the regular and recognizable $1-2-\mathrm{Hz}$ delta wave we associate with the global noradrenaline signaling at around 18 months of age in human infants is, in the present model, a result of the onset of consciously controlled activity (with variable intraepisode attention spans), the onset into coordination of previously dissociated and habitually automated, prolonged multipleepisode activities that possibly override the primary and more general percept-action cycling, or more likely, both of these. After 18 months, episodic time scales and blink rates are highly variable, reflecting conscious cognitive activity. The diminution with senility in the capacity either to exhibit conscious attentional control or to execute coherent multiple-episode activity allows re-emergence from the apparent EEG noise of a now depressingly regular delta wave, indicative of regression to the primitive percept-action cycle.

So we are led to predict a tight logical, functional, physiological, and neurochemical connection in the activity of the patches of the striatum, the amygdala, the locus coeruleus, the climbing fibers of the cerebellum, and the eyeblink.

The gating of physiological activity throughout the brain to discretize experience and capture episodes of experience in memory is normally flagged by the eyeblink. Under normal conditions, no eyeblink, no memory formation. Without the gating underlying these, no eventually higher human competence can arise: coniveo ergo sum (eyeblink therefore I am).

\section{CAPACITY TO DEAL WITH UNCERTAINTY}

If the eyeblink rate at $1-2 / \mathrm{sec}$ is correct for episodic gating, then a human lifetime might correspond to $5 \times 10^{8}$ waking episodes in an unpredictable environment. There is an initial multiple-salience requirement of episodes to support sensory submodal feature dissociation, but this is a transient phase. If each of the six senses is nominally ascribed 10 articulable and storable perceptual features (including a 'null'), then each episode could be characterized by above-threshold information in $10^{6}$ ways once all the sensory submodalities had become dissociable, so that the brain could selectively respond to capture $5 \times 10^{14}$ episodes of different saliency. With an endowment of $5 \times 10^{10}$ neurons, but some $10^{14}$ synapses, we must then lean toward Hebb's (1949) insight that the synapse rather than the neuron is the most fundamental memory unit. Rules for elemental synaptic plasticity are already being explored (Levy \& Desmond, 1985a, 1985b) but it is clear that understanding of a single synaptic system is of limited utility (Gazzaniga, 1986).

The human brain is thus adapted to deal with the moment-to-moment uncertainty of the perceptual environment through an entire lifetime, even if episodic processing actually involves such slow cycles of the order of seconds, as is proposed. However, this potential for the brain to deal constructively with uncertainty can only be realized through learning, which involves a noise-rejection filter of triangulated episode comparison. This leads to a fundamental and unavoidable temporal structure in the development of learning, beginning with innate and subconscious perceptual learning (to 'feature-extract' and 'objectify,' which is based upon modal dissociation, then progressive submodal dissociation) and proceeding on to conscious behaviors and thought.

\section{THE ORIGIN OF CONSCIOUSNESS}

While having insisted on the subconscious learning-byrecurrence mechanism that articulates features of Type 1 or Type 2 objects through the first three behavior classes, it will be clear from the character of the three higher observable classes of behavior (covering skillful activity, concentration, and deliberate action) that some overlap with the general notion of consciousness has crept in to the characterization of later behavior. The very use of words such as 'choice' or 'deliberate' connotes evaluation across known alternatives: this is the essence of conscious activity. Just how does the system, operating entirely subconsciously to objectify perceptual objects from the environment-the 'system' essentially described in the first paper-become transformed to have the hallmarks of conscious behavior?

It seems reasonable to seed this transition in the onset of availability of general inhibition in both the perceptual and the action domains. Class 4 and Class 5 behaviors provide for selective attention while action proceeds or is inhibited, respectively. This inhibition of general action or of general perception probably does not come naturally to the early learner; it is almost certainly a capacity stimulated by external agency.

Explanation of this point touches on the whole panoply of agent-learner interactive learning, on language utilization, and on the role of symbolism generally in human behavior. This explanation will compel us to the view that personal consciousness, as awareness of alternatives copresent, together with a capacity for choice in action, is explicitly of social origin.

Our analysis of learning and behavior so far provides us with the constructs to undertake this explanation with 
reasonable economy, provided we first backtrack to a particular aspect of early conceptual learning touched on in the first paper. This concerns the meaningful contrivance of association between perceptually disjoint objects, objects that under normal perceptual learning processes would become dissociated rather than associated.

The characterization of this process as articulated in the previous paper was first in terms of early language learning, when names are attributed to objects. Later we alluded to subsequent passage (through number learning) to the point when more general symbols could be understood to represent (and not merely be a perceptual attribute of) designated objects (e.g., Seabourne, 1975). In the first paper, the possibility of failure in attempts at contrived conceptual learning was identified; this possibility was claimed to be realized in autistic children, in that they lacked the understanding of this bidirectional equivalence in symbolic representation, leading to their lack of facility with the dual role of spoken language.

While not wishing to detract from the prominent role of language in higher behavior, naming is merely an example of more general symbolic signification. Symbolism probably begins with gesture (Acredolo \& Goodwyn, 1988; Hewes, 1973; Noble, 1987), and goes on to occupy the center stage of all intellectual activity (Church, 1956; Frege, 1964; Langer, 1967) as an essential ingredient in conceptual objectification, which is inescapably part of the human condition.

Seemingly, man is compelled to objectify even the most ephemeral of objects (e.g., in the symbolization of ingredients of dreams; see Jung, 1964/1978)-though always on the basis of recurrences and polymodal analogy. The capacity for drawing conscious polymodal analogy across Type 1 (or later, Type 2) objects derives contingently from the fundamental episode-comparison logic which subconsciously determines 'mutual relevance' between episodes. It is this consciously 'analogic' power of the human mind that is developed very shortly after the onset of the most primitive forms of conceptual learning which provides the basis of the development of higher order thinking skills (Friedman, 1984; Galton, 1870).

The foundation for the onset of conceptual learning is that it depends on contrivance by an external agent to initiate the linking of perceptually disjoint objects. The example of a physical object < apple > and an auditory object < "apple" > has been presented as objects that share no attributes. The question arises as to why this should be difficult for the learner, and why it should require the intervention of an external agent at all.

The first point is that, apart from the startle reflex, the early behavior of infants is never characterized by marked general inhibition until the onset of locomotion. The word "no!" first becomes repeatedly heard at this time; it becomes one of the earliest words in the infant's vocabulary. Prior to this stage, the infant is engaged in what we have termed ticking-over behavior. This nonproblematic meandering activity characteristic of a child's play and initially unsupported by attention or skilled procedures is the basis of sensorimotor learning. These behaviors are freely voluntary and are characterized by a continuous meander through the perceptual environment.

In an important paper, Libet, Gleeson, Wright, and Pearl (1983) reported assessments of the reportable time (W) of awareness of conscious intention to freely act, in relation to the onset of cerebral activity monitored as the 'readiness potential' (RP) in EEG recordings. They used electromyogram (EMG) recordings as indicative of the actual time of a voluntary motor act. The recordable cerebral activity (RP) that precedes a freely voluntary, fully endogenous motor act was directly compared with the reportable time $(W)$ for the appearance of the subjective experience of 'wanting' or intending to act.

The onset of cerebral activity clearly preceded the reported time of conscious intention to act by at least several hundred milliseconds. Libet et al. (1983) concluded that the cerebral initiation of a spontaneous, freely voluntary act can begin unconsciously-that is, well before there is any (at least recallable) subjective awareness that a 'decision' to act has been made. If generally true, this would introduce profound constraints on the potential for conscious initiation and control of voluntary acts. ${ }^{43}$

However, conscious veto of an unconsciously initiated act is possible "because reportable conscious intention, even though it appeared distinctly later than onset of RP, did appear a substantial time (about 150 to $200 \mathrm{~ms}$ ) before the beginning of the [actual] movement as signalled by the EMG"' (Libet et al., 1983, p. 641).

A general hypothesis had al ready been proposed [by Libet] that some substantial time period of appropriate cerebral activity may be required for eliciting all specific conscious experiences. This developed out of experimentally based findings that cortical activities must persist for up to $500 \mathrm{~ms}$ or more before 'neuronal adequacy' for a conscious sensory experience is achieved. In that hypothesis, those cerebral activities that did not persist sufficiently would remain at unconscious levels. The present evidence appears to provide support for that more general hypothesis. It suggests that a similar substantial period of cerebral activity may also be required to achieve 'neuronal adequacy' for an experience of conscious intention or desire to perform a voluntary act. (Libet et al., 1983, p. 640)

Libet et al.'s (1983) paper represents strong evidence that the ticking-over mode of behavior is subconsciously mediated and cannot be interrupted without explicit conscious intervention. Why would a child come to consciously intervene in natural, playful behavior? In the case of two objects in the perceptual environment, whether perceptually disjoint or not, surely the child will explore these objects sequentially, attending to and playing with each in turn.

In fact, the play of the child must be interrupted to bring any two such objects to the child's attention. The caretaker intervenes and explicitly teaches the child to attend simultaneously to two disparate objects. This occurs in two general cases (see previous paper), first where objects are perceptually disjoint, as in naming, and second where objects are perceptually similar, in which case they are counted. In the latter case, two perceptually similar and 
familiar object types that are spatially distinct are considered simultaneously as a named group < "two" >

The child's natural instinct in either case would be to consider each individual object as separate. Only at the (gentle) insistence of the caretaker, ${ }^{44}$ through repeated presentations of the perceptually or spatially disjoint objects in temporal contiguity, does the child learn to inhibit the naturally sequential scanning habits of play.

The process by which this occurs is an externally mediated biofeedback, using the internal mechanisms already available-in Class 2 and Class 3 early behaviors - to alter phasic onset and duration of elementary percept and actual elements. Using only these skills, the child can modify the natural and spontaneous phase relationships of readiness potential, awareness, and actual movement. In fact, the internal inhibition of movement-contrived or prompted by the caretaker (<"no!" >) -is achieved by the child learning to defer the actual onset of the readiness potential for a movement into simultaneity with the conscious awareness of intention to move. Further learned deferment, and ultimately the negation of the movement as well, can follow, leading as we have seen to the availability of inaction in Class 5 and Class 6 behaviors.

The re-presentation of the perceptually disjoint, or spatially disjoint, objects by a caretaker provides the fundamental experiential basis of temporally simultaneous object awareness for a child, and inaugurates a systematic back-and-forth scanning of the separate objects within a single episode. Such back-and-forth scanning of cotemporal objects is made possible by intraepisode saccadic processing and is familiar in adult perceptual processing, but for the child the second case of spatially disjoint but similar objects (as in counting) is derivative and dependent on the prior successful naming of one of the objects. Since this precursory phase requires a visual or spoken gesture (a Type 2 object in perception) to be linked to a polymodal Type 1 object, one presumes that such linking is nontrivial unless the temporal extent of the Type 2 object is limited in duration to be-at least in early learningwithin the span of a single episode. That is, names (or equivalent gestures as in pointing) should be short.

With externally contrived biofeedback designed so that the infant will consider and reconsider co-presented objects, one has the fundamental devices upon which internally constructed conceptual objects depend. First, the concept is of no value until it is named-thus, naming provides the first meaningful attribute of a concept in early learning-but even then the concept of an object cannot be formed without consideration of at least a second and distinct extension of the concept (as in counting). ${ }^{45}$

After experiencing several instances of copresentation of two independent objects, the experience of back-andforth sequential scanning of the two gives over to simultaneous perception of the two independent elements as two objects that have independent existence, but nevertheless co-occur. The repeated co-occurrence of the two perceptual objects makes each equally meaningful, and the subsequent appearance of one alone can stimulate the search for another, typically co-occurring, object of past experience.

So the child learns through these experiences that there is an element of choice in the order of perceptual scanning in such familiar circumstances. It is the availability of choice, together with the deliberate, concentrated scanning of the perceptual environment for objects that are expected on the basis of those already present, which is the hallmark of conscious behavior.

Assuming that such behavior cannot arise spontaneously [taking the results of Libet et al. (1983) to be general for freely voluntary acts], the element of choice must be learned through the external agent's intervention in conceptual learning and is thus socially derived. Conscious behavior will not be exhibited without the enabling stage of contrived conceptual learning. The teaching of language use in naming, and later in counting, gives rise to internal concept formation but the discipline of consciousness arises from the teaching process itself.

\section{THE LOCALIZING ROLE OF LANGUAGE}

'Thought,' where in general the motor system is inhibited, subsumes this capacity for choice. Recognizing its anticipatory character, we can identify two of thought's distinguishing attributes, as is schematized in Figure 15 in terms of virtual action.

The learned capacity to anticipate the outcome of a familiar motor transform comprises the second major aspect of the activity we call thought, so we may now integrate thinking into our taxonomy of percept-action configurations (see Figure 16). However, this initial portrayal of thought is still incomplete, because it does not account for the symbolic element of thought. Figure 16 conveys the perceptual anticipation, but not the almost universal verbal mediation of thought. Churchland (1986, p. 379) provides a hint in her recounting of an excellent example of people's behavior in a building in response to <"fire!" > A broad variety of perceptual cues (the sounding of an alarm, a whiff of smoke, the appearance of flame) will elicit movement toward an exit, but every possible primary cue seems logically to channel to a single verbal symbol <"fire">, which encompasses the variety of possible primary stimuli, and which itself becomes the specific and localized cerebral cue for embarking on avoidance action, whatever the modality of the initial sense of danger. The adaptive significance of tagging the onset of avoidance action to a simple and universal sign, rather than separately to each possible primary prompt, is obvious from a complexity viewpoint.

Here we begin to appreciate the wider application for the intensional character of verbal reference arising from primitive conceptual learning. Not only can the perceptually disjoint label for an object serve as a general designation for a class of objects, but a simple perceptually based symbol can serve as an 'interrupt' to general percept-action processing. Furthermore, specific behavioral activities can be instigated, through learning, in 


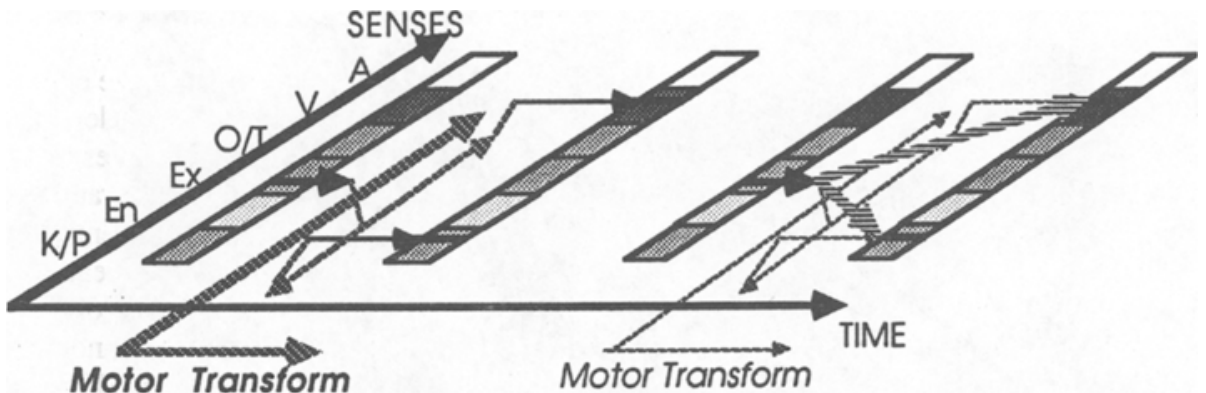

Figure 15. Virtual action. The capacity to anticipate the outcome of a motor transform is required to support any programming of perceptual attention. This requires, in turn, that the motor transform be familiar - that is, that the underlying percept-action cycle has already been learned through repeated instances. Becoming familiar with the perceptual outcome of a motor transform creates the possibility of anticipation of that outcome given an initiating perceptual context. Anticipation of the outcome of a familiar motor transform in a given context affords the possibility of perceptual processing sequences as though intervening motor activity had occurred. The anticipation of the outcome of a familiar motor transform in the absence of its occurrence (possibly through active inhibition of its activation in the perceptual context) constitutes virtual action. The possibility of virtual action is adaptive because the screening of possible action alternatives can proceed without their explicit temporally extended actuation. This requires one episode per Type 2 object, regardless of the real-time duration of the Type 2 object were it activated.

response to the symbol regardless of all other features of the current perceptual environment.

At the next higher level, the invocation of one (e.g., verbal) symbol may, again through experience and learning, become a selective prompt to 'imagine' or anticipate a second (e.g., verbal) symbol. In this case, the second symbol becomes the prompt to physiologically activate its familiar perceptual referents, which correspond to familiar extensionally supported perceptual registrations from the environment when confronting the object/concept designated by the word. However, if the activation is too strong, it might be distracting. Thus, the learner must learn to control the activation so that it is activated as a background while retaining a primary locus of activation

\section{Class 7: Thinking}

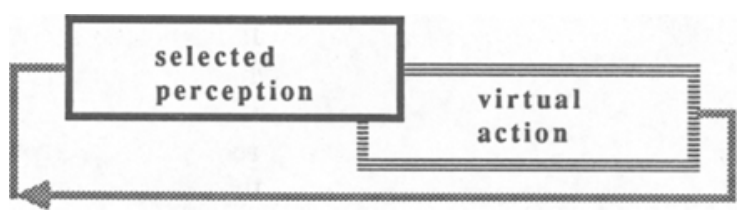

Correlate: Global action inhibition,
with selected 'as though' virtual
action to generate previously
experienced perceptual transform

Figure 16. Class 7 behavior (thinking). Thinking is a refinement of Class 6 behavior that encompasses all the elements of Class 6 behavior except that the motor system is globally inactivated while screening of alternative percept -virtual action sequences proceeds. in the cortical location associated with the perceptual features of the symbol rather than its referents.

We see a focusing referential system in the use of language that enables one to tag action to localized rather than delocalized cortical activity. The meaningfulness of any such symbolic label naturally depends on the breadth of experience of different contexts in which the label is appropriate, so that the complexity of circumstances that can activate a 'word' in thought, or the complexity of possible perceptual elements that can be activated by 'bringing to mind' a word grows with actual experience.

In normal verbally mediated thought, we might expect the use of verbal symbolism to correspond to a concentration of cerebral activity in Broca's area and/or Wernicke's area of the cortex. In general, the modal/submodal discreteness of the normal mode of symbolism serves to localize actual cerebral activity specifically and predominantly to the corresponding modality at the cortical level. Note how these ideas smoothly mesh with the modal similarity criterion for local trajectory 'following' in Type 2 perceptual learning discussed in the previous paper.

In Figure 17, an attempt is made to symbolize elements of the dual localizing and activating character of verbal behavior, and of symbol-based thoughtful activity in general. The actual perceptual modality of the symbolism is logically irrelevant; it could be verbal, tactile, gestural, or visual in origin. The utility of symbolism lies only in its perceptual discreteness and simplicity together with its continuing disjointness from its referents.

This makes clear the adaptive significance of symbolic thought localized through partial dissociation of the symbol from its referents, giving associated preferential localization of cortical activation (Figure 18) in a thought sequence. The basic process that underlies it-the generation 


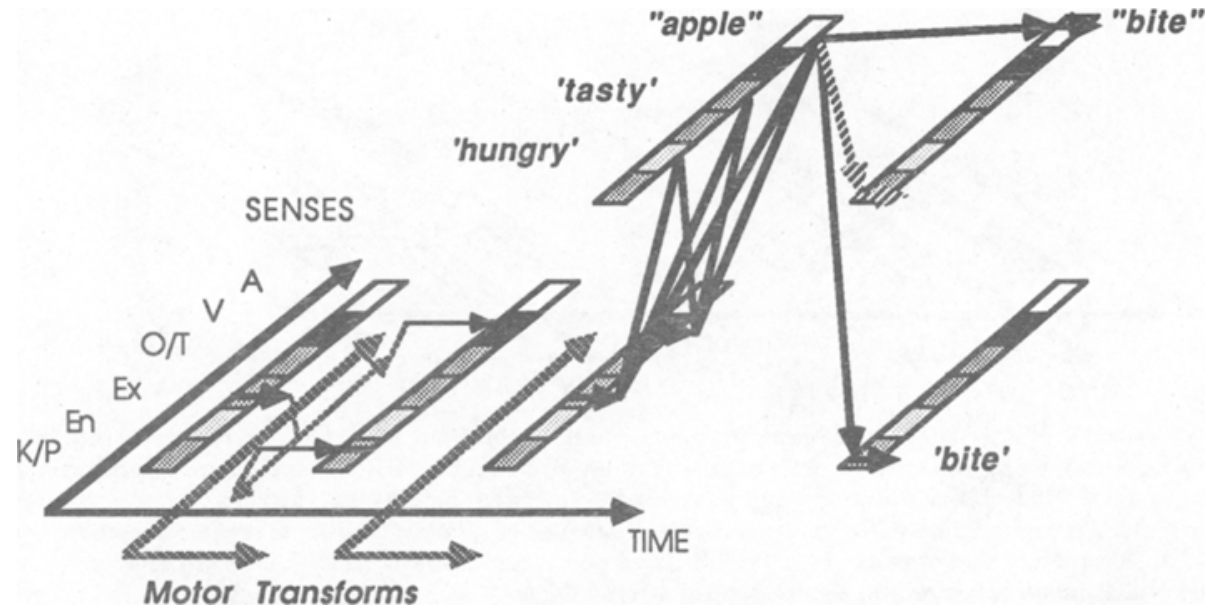

Figure 17. Symbolic thought. Symbolic, often verbally mediated thought serves as a vehicle for localization of cerebral activity to become predominant. 'Left-brain' dominance in normal verbally mediated thought arises in this manner, but would be manifested as differently localized focal activity should tactile (as in Braille in the case of the deaf/blind) or visual (as in the case of sign language for the deaf) symbolism be the basis of the symbolic tagging of concepts.

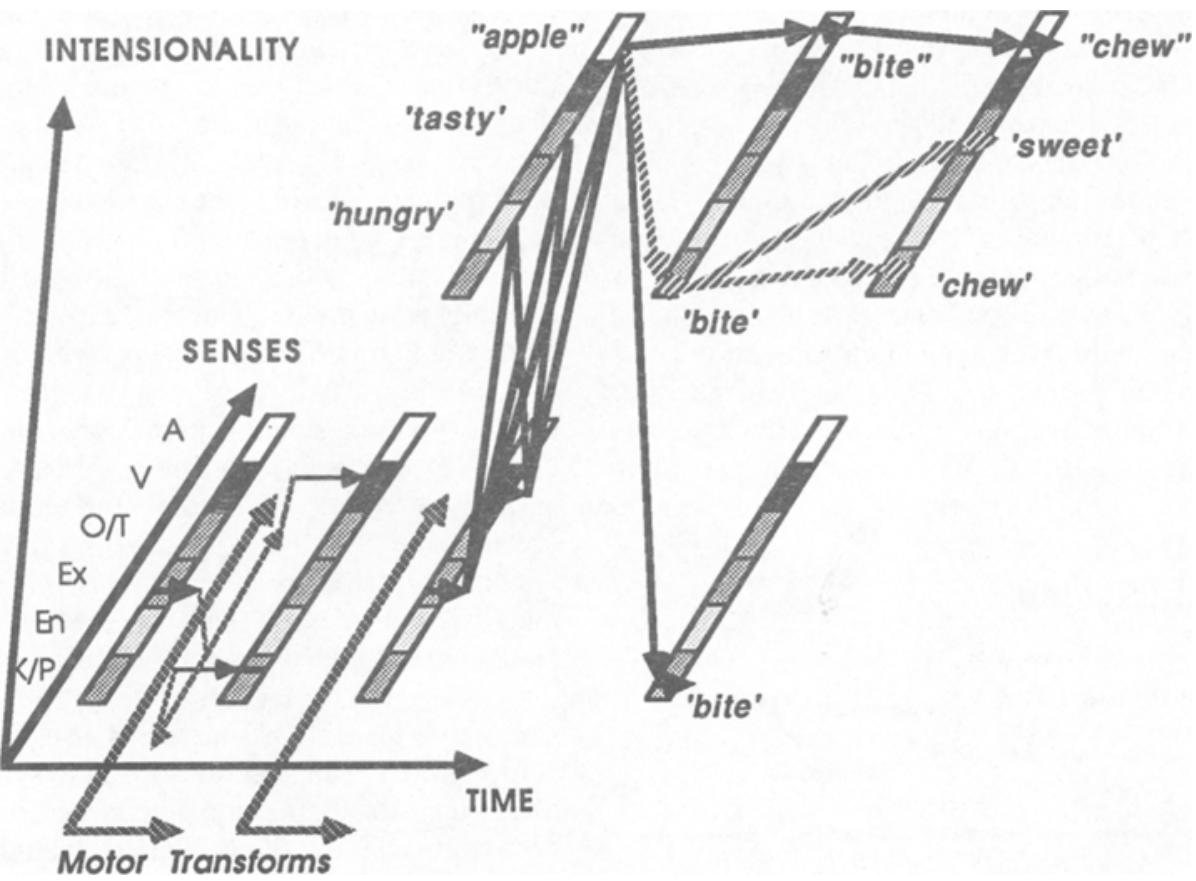

Figure 18. Symbolic thought and cerebrally localized neural activation. Creative artists may think in colors, sculptors in shapes, choreographers in movements; these are examples of impressionistic, primitive Type 1 thought, which should be localized differently than verbally mediated thought. Symbolic, often verbally mediated thought serves as a vehicle for localization of cerebral activity to become predominant in the Broca/Wernicke complexes, but cerebral localization of activation depends on the sensory modal basis of the symbolic label used. An example of Type 2 thought is anticipating others' reaction to one's behavior, the subject matter of ethics and morals, which is likely to be localized in the (pre)frontal cortices. Without the preferential localization due to symbolic thought, as shown in the upper right portion of this figure, thought sequences would wander all over the cortex, rather than through a more locally accessible succession of symbols, which, while retaining weak association to their referents, span a limited submodality range. 
of links between disjoint perceptual objects-remains the general enabling mechanism that must be experienced, but thought is greatly expanded in scope and significance when it is unconstrained by what an external agent may contrive. Autonomously generated conceptual links between contiguous familiar objects provides this expansion.

Familiar objects, once they have become familiar, and once the process of symbolic tagging itself has become familiar in language learning, can also become relinked with environmental cues that are disjoint from the objects themselves but are associated with the episodic contextsor later the events-in which the objects have occurred. Objects can thus serve as the basis of event recognition and recall. Event recognition, which arises through a complementary Type 2 partial dissociation of temporal tags previously associated with an object, is seen to mirror the Type 1 partial dissociation of the symbolic label to an object from the object itself. Event recognition, and the later objectification of the time dimension, are thus portrayed as highly derivative forms of learning.

So we see that some important and significant categories of learning are enabled by means of the process of association, but every category of learning gets its refinement only by dissociation, in one way or another.

\section{TICKING OVER VERSUS PROBLEM SOLVING IN LEARNING}

It is intriguing to reflect on which of the different overlapping and interdependent categories of ordinary behavior might be most directly implicated in learning. The perceptual dissociation and objectification model of learning as it has so far been expounded could draw its major implementation from (1) problem-solving behavior, (2) active (intentional) behavior, or (3) the vast preponderance of our ordinary behavior, which is neither particularly active nor problematic, at least in the consciously purposeful sense.

I consider this third category, which I call simply 'ticking-over' behavior, to be something of a Cinderella in the learning literature, in that it has been too long overlooked. Consideration of the first two categories of behavior has dominated the psychological and educational literature for at least a century, despite the fact that the majority of our waking lives is spent in the ticking-over mode rather than in either of the other two modes. Is all this time simply wasted?

If one explores the possible significance of the tickingover mode in the context of the present learning theory, it is readily recognized that the data-driven character of the episode-processing scheme allows all of our waking behavior to be supportive of the acquisition of stored episodes of polymodal perceptual experience. This includes the ticking-over mode as well as the characteristically conscious problem-solving mode and the purposefully active mode.

Unfortunately, the paramount role played by conscious activity in social communication has led to the erroneous assumption that conscious activity plays a uniformly para- mount role in other kinds of behavior, particularly those that support learning. This has certainly compounded the mystery surrounding selective behavior generation, which is, for the most part of our ordinary experience, anything but conscious: most of our behavior-trajectory changes are rapidly and imperceptibly generated well below the level of fully conscious intervention.

Rather than embellishing the putative role of conscious behavior in support of learning and selective behavior generation, we must instead explore the radical alternative, that conscious activity is merely a precondition for certain limited (though ultimately powerful) kinds of learning. In the extreme version, we could relegate conscious activity and its cultural epitome, rational behavior, to a purely communicative significance.

Given our assumption that autonomous learning is both logically possible and a practical reality, the corollary must be that communication is inessential for every category of learning. I have already implicated direct (spoken, gestural, or demonstrative) communication from an external agent to a learner in at least the early stages of conceptual learning. We can admit a similar role for indirect communication (e.g., in the form of the written word or even through a culturally structured environment) in much of the mature learner's learning. However, it cannot be supported that every category (or even the predominance) of learning is similarly dependent on communication. This is surely not the case for autonomous perceptual learning, which objectifies segments of the continuing perceptual influx. If learning in all categories is supported by the same basic computational logic, and if perceptual learning is indeed autonomously possible, then communication simply enables certain categories of learning rather than being an essential component in the primitive mechanism of learning.

In fact, we arrive at the even more radical view that rational behavior, or any other form of consciously purposeful activity, is not only unnecessary for all categories of learning, but even for the categories of learning for which such activity is enabling or prerequisite; learning is actually inhibited for the productive interim of such activity. This has profound implications for the folklore of learning, because even thinking, as a category of conscious activity, becomes simply a review of learned information rather than a correlate or the source or the architect of learning itself.

\section{THINKING AND NONTHINKING SKILLS: DECISION VERSUS JUDGMENT}

Once communication is proposed to be temporally disjoint from learning and the role of conscious or rational activity is called into question as the key ingredient in learning, we are led to appreciate the significant distinction between the consciously rational pattern of analysis leading to a decision concerning future activity on the one hand, and the rapid and subliminal achievement of a perceptually stimulated judgment on the other. We can discriminate between these two types of decision in the fol- 
lowing terms: If the former process of decision making is temporally extended and of consciously overt character, then one can characterize the reaching of a judgment as typically subconscious and rapid (in fact, intraepisodic) on the episodic time scale.

The activation of specific and selective behavior, often in the form of communication aimed at conveying the result of a decision or a judgment, typically ensues following completion of either process. Whatever path may have been followed to the decision or judgment point, the rigors of communication require a rationalization of the antecedent process in formally communicable, serial terms. In this view of ensuing communication, either antecedent process of decision or judgment elicits similar behavior. However, the processes themselves are quite different and have been explored in a very imbalanced manner in the literature, with rational decision making the subject of the entire discipline of logic, while judgment, at least in the specific context that is proposed here, seems to have been almost entirely overlooked, at least in the formal literature.

Given the vacuum surrounding the judgment process, I propose to link the judgment process squarely with the contemporaneous underlying process of episode comparison. This confers on judgment the character of mediation of the outcome of the comparison of mutually relevant episodes that have both commonalities and differences. The outcome of such a judgment process is almost invariably associated with the (selective) activation of motor activity of one kind or another. Thus, one may speak of the judgment process mediating the transition between percept (a perceptually registered and storable episode of experience) and action (in the form of selective activation of the motor processors currently accessible). We have already elaborated the formal process of episode comparison by the explicit addition of some internally activated motor transform interspersed between each successive pair of episodes.

\section{GENERAL LEARNING OPTIMIZATION}

If one were to admit the possibility that stored episodic traces are subject to slow degradation (or to interference through nonuniqueness of sensory-information traffic associated with different objects), then the likelihood of exceeding the relevance-threshold match condition against stored episodic information in a given context would probably depend upon the recency of the previously stored episode.

In addition, if learning is exemplified by the selective elaboration of previous episode-vector traces in a way dependent on the structure of the overlap with the currently registered episodic vector (i.e., if selective behavior is indeed activated on the basis of the structure of the overlap between the current episode and an earlier-real or virtual-relevant episode), and if one admits to the impressionistic nature of the earliest prior perceptual registrations of a new domain, then the directionality of action or selective attention across the modalities is quite un- predictable early in experience with any new domain. In this instance, providing complete control in the directedness of action to the learner would probably enhance learning by means of immediate feedback to internally generated action (if, of course, the action repertorie is already sufficiently broad).

Moreover, learning should be optimized by contriving the learning environment so that the learner can access all corners of the domain in any sequence, since no observer can predict the early constrained but unknowable trajectory of learning.

Thus, recency, control, and access are three criteria for optimized learning environments. The learner's trajectory in the learning environment can be monitored by an observer through the apparent evolution of the learner from a subjective to an objective state, by the learner's evaluation of the environment so that function can progress from cognition to direction, and by observing the adaptive process in each new environment where behavior begins with invention and ends in control within the domain.

These ideas apply generally across every category of learning conforming to the psychonomic logic-that is, in perceptual learning, in conceptual learning, and in skill development. In perceptual learning, learning is facilitated by structuring the perceptual environment so that objects of learning recur in similar or familiar contexts, which nevertheless exhibit gradual variation rather than remaining static.

In conceptual learning, as to some extent in skill development, one can see a formal contrast with perceptual learning through the optimizing role of 'neg-instances' (Englemann \& Carnine, 1982), since they provide general constraints (in concept development) or explicit constraints by time-step (in skill development) that are useful even before the concept or skill is completely articulated and fully reflected in capacity for thought and action.

Involvement of a tutor facilitates the appreciation of neginstances in conceptual learning, and through modeling and demonstration in skill development a tutor promotes the selective refinement of appropriate motor activities

Table 2

Optimization Strategies for Teaching from the Psychonomic Theory

Use a familiar environmental context for learning

Provide a holistic view of domain to begin each lesson

Avoid distractions to concentrate on target domain

Structure novelty into lessons from a familiar base

Demonstrate components of required skills

Explicitly guide pupils through each new procedure Avoid substantive repetitions without selected modification Use reviews to highlight important aspects of the domain

Allow each pupil control of his/her own learning Encourage active learning engagement by each pupil Let each pupil complete his/her own learning segment

Provide explicit labels for important objects and procedures Provide multiple paths to important features of the domain

Provide open access to the domain for exploration

Switch learning domains cleanly

Allow for relearning in the context of a new domain 
supporting skilled performance. In either case, contrivance by an external agent can establish the intrinsic learning path that leads to internally constructed knowledge in a (culturally) desired, rather than a random, direction. The ingredients of the external agent's activity that will have the most impact on the learner will comprise (1) appropriate paradigm setting and (2) guided retraversal of each activity in a similar setting.

Review of the learning principles arising from the psychonomic theory suggests general optimization strategies in formal approaches to the teaching process, as shown in Table 2.

With Montessori teaching methods, recall is always focused on the start of any activity sequence. The psychonomic theory outlined above suggests a theoretical foundation to such teaching practices, in that the activation of any partially learned sequence or partially automated general procedure can only occur in the context of a discretely episodic system of choice, and choice can only access, within the intraepisodic judgment process, the beginnings of future actions.

Self-management skills will be enhanced by the orchestration of a readily accessible 'header' to the data structure for any action procedure, whereby the circumstances and contingencies appropriate to invocation of a specific procedure from the available repertoire are clearly articulated; this will facilitate the initiation of the 'most appropriate' selected procedure. Furthermore, it is highly probable that a generalization of the virtual action concept is applicable here, in that the selection of the most suitable procedure from the available repertoire would be aided by the augmentation of the procedure header with some articulated schema related to prospective consequences emanating from that procedure, so that some form of 'procedure-consequence' to 'current goal' match can be made in anticipation of the procedure being initiated. Ideally, any procedure will be activated only following both a good match between 'current circumstances' and 'initiating circumstances' for the procedure and a good match between 'current goal' and 'likely consequences' of the procedure. On the basis of experience, should some symbolic consequence-of-procedure information be telescoped into the initially accessible procedure header, then it may be unnecessary for the procedure to be followed through step by step to completion before some idea is available of the likely outcome of activation of the procedure. Contriving facilitation of learning of this kind may promote more efficient learning and the more rapid growth of competence by means of the minimization of fruitless activity. This is just what parents and teachers are actually doing when they seek to build 'character' into each child.

Finally, some interesting implications exist for our extreme radical view of the role of consciously purposeful activity in personal learning. If it is indeed the case that such activity is associated with either an inhibition or the preclusion of learning for the duration of that activity, then in order to optimize learning in a novel and unfamiliar environment about which little could be predicted, one should deliberately restrict the intrusion of consciously rational behavior in the early stages of learning on the grounds that it simply displaces activity that can directly support later learning. That is, one should relax instead into the simply and reliably acquisitive ticking-over mode to allow the most rapid possible acquisition of a wide variety of episodes that effectively span the whole domain. ${ }^{46}$

Such an acquisitive strategy begins as eclecticism constrained only to the general domain. Accepting our behavior-generation-through-structure-of-episode-overlap hypothesis as a fundamentally subconscious process that is not amenable to conscious scrutiny, one should further refrain from the imposition of any artificially constrained trajectory in early learning in a new domain and simply let the intrinsic learning capacity inherent in the episodecomparison/behavior-generation logic directly guide the learning trajectory.

Autonomous learning will inevitably be domain-specific, except to the extent that domains are incompletely disjoint, when the structure in the intersection of the domains may allow or constrain the transition from one domain to the other in the absence of contrivance by an external agent.

The psychonomic theory presented here renders the evidently chaotic and unpredictable course of personal learning comprehensible because most of the work is done subconsciously. Thus, the theory makes it comprehensible that experts are typically unaware how they actually become expert.

The theory makes essentially no demand on the temporal structure of learning in any domain beyond the requirement that mutually relevant episodes be eventually acquired. We can, however, be sure that extended episode sequences in a domain will provide the optimal support for effective subsequent learning if the domain or general context in which specific knowledge or competence is sought is 'switched into' and 'switched out from' as cleanly as possible when moving between disjoint (and therefore mutually unproductive) domains. We readily see that maintaining a general focus on a learning goal in a particular domain, while keeping in mind matters pertinent to a disjoint domain, must reduce learning efficiency. Accordingly, complete concentration in the selected domain while switched into that domain must enhance the prospects of learning, especially if it is uncontaminated by inappropriate constraints on the learning trajectory. ${ }^{47}$ Thus, we imagine that an optimal strategy for long-term productivity in learning is to ensure sharp breaks in the transition from one context to the next; that is, rapid and complete context switching should be advantageous. In consequence, various neural, emotional, or behavioral deficits associated with either facile distraction or obsessive behavior mitigate against the possibility of optimal learning unless some compensating selective-attention self-programming can be learned.

The structured irrationality concept for optimal learning early in a domain is in accord with the general observation expressed by Halford (1982), who held that no specific training procedure has ever been demonstrated to have clear superiority over any other in cognitive learn- 
ing. Those who are unhappy with structured irrationality in their early-learning trajectory in a new domain will no doubt be willing to articulate a more effective optimization strategy for the acquisition of useful personal knowledge.

\section{BROAD-CONTEXT AND OJECTIVE REPRESENTATIONS}

According to the psychonomic theory, the fundamental operational basis of the brain rests upon encodings (Lashley, 1963; Hinton, 1986), which are polymodal information segments obtained in the form of episodic gatings of experience. From these polymodal information segments, a process of dissociative learning partially abstracts objects from the broad episodic context in which they occur. This serves to support the acquisition of declarative knowledge of both Type 1 object intensions (concerning the things of the environment) and Type 2 object intensions (concerning dynamically varying activity perceived in, or enacted upon, the environment).

Any Type 2 action (or prolonged activity) object must be initiated in an episodic time frame, and the Type 1 episodic context in which such a Type 2 object is launched serves in a later but similar context as the prime for subsequent activations of that or a similar object. In this way, the Type 2 activity depends upon its Type 1 generating context.

This embellishes the static perceptual attributes of the typical Type 1 object with dynamic attributes associated with the activity that can be generated from (or stimulated internally by) that object. This conveys a projective character to the initiating Type 1 object. In fact, every Type 1 object acquires at least some 'callable' dynamic attributes that can project the learner in selective ways through the environment.

Humans become programmed in an object-oriented fashion (Meyer, 1988). In general, every learned internal Type 1 object will automatically stimulate its most salient action in the current context unless the learned choice mechanism of consciousness intervenes.

\section{CONCLUSION}

Many of the questions raised at the outset of the first paper have been touched upon in the presentation and initial defense of the psychonomic theory, but clearly definitive answers concerning most of these questions will depend on the outcome of experiments that have yet to be performed. It is hoped that even in its present rather general form, the theory will stimulate some interesting new approaches to the design and execution of relevant experiments in fields from neuroscience to education.

The theory may also provide a new perspective from which to review some perennial questions. For example, in the field of artificial intelligence, the present way of thinking of the brain's operation provides a new insight into the problem of machine synthesis of 'intelligent' behavior, since the psychonomic framework rests upon an extremely simple rule structure and complex polymodal, full-context data structures rather than the converse, and has action adaptively coupled to percept, eschewing static knowledge. Second, some new insight into the Aristotelian notion of abstraction has been developed, which makes the natural intensional data structures of the mind far less readily capturable in machine logic than has been commonly supposed so far. Conventional computers must give way to 'connection' machines in order to simulate natural logic efficiently.

To give another example, and without getting too involved in philosophical matters here, one cannot help but align this kind of theory with that espoused by Jacques Maritain on 'existence' (see Devine, Held, Vinson, \& Walsh, 1986, p. 363), despite the weight of opinion against such a philosophy.

It now rests with each reader to review his or her own issues-and-constraints checklist, along with his or her own store of expert declarative knowledge, to assess the degree to which the psychonomic theory presented here actually accords with all the facts. We can say that it seems to map well onto the actual process of the development of behavioral competence from infancy (e.g., see Appleton, Clifton, \& Goldberg, 1975; Ilg \& Ames, 1955; Matlin, 1983) and the actual development of language utilization from childhood (e.g., see Bloom, 1975; Garrett, 1982; Hill, 1986; Hill \& Arbib, 1984; Peters, 1983), but this is more an amateur than an expert judgment. In any case, detailed review of the full relevance of the psychonomic theory to child development is not possible here for space reasons. We can only reiterate that in the psychonomic theory no knowledge is required to be innate, and state as a precaution that the important role of motivation and drive in learning has been included by the simple device of relegating emotions to be merely one of the six senses (viz. endoception). Because most learning is construed as not only motiveless, but subconscious, emotions simply take their ordinary place in the spectrum of the six senses.

The first paper outlined a hypothesis of the logical basis of learning, as a fundamentally dissociative process operating over global polymodal gating of the brain's perceptual acquisition systems. On the basis of that hypothesis, in the present paper a framework has been developed for the logical basis of the development of human behavioral competence. The five foundation assumptions of the theoretical framework (see Appendix) - the single episodecomparison learning rule and its attendant axioms in the theory, the learning by differentiation according to the triangulation logic, the universal data structure concept ranging over two temporally distinguished types of object intension, the derivative but deeply enabling role of association-representation learning, the three categories of knowledge distinguished by content in the various acquirable data structures, and the taxonomic distinction of seven classes of behavior (including six learnable types)-all now require independent scrutiny, as does the coherence, consistency, and completeness of the entire presentation.

The psychonomic theory attributes plausible functionality to explicit anatomical structures, neurophysiological connections, and certain neurotransmitter systems, and 
has led in particular to an explicit prediction concerning the combined role-and close temporal interrelationship in processing - of the striatum, amygdala, locus coeruleus, cerebellar climbing fibers, and the eyeblink in association with global episodic gating. Any real-time interrelationship of functionality between these contributors to the brain's basic function remains to be established, hopefully guided by this theory.

The present discussion has dealt only with the most primitive forms of learning in each category, and with the general principles of selective behavior generation. However, it is clear that the range of alternate possibilities for selective attention/action based upon a number of viable strong overlap but changed perspective segments in the episodic vector mutual relevance assessment is the key to a lack of determinism in the perception-action cycle. Consciousness itself is directly dependent on the existence of such alternatives, but a default mechanism of choice-making of the subconscious kind must also operate to always yield one action alternative. This subconscious choice-making is typified by the instantaneous judgments that are the basis of our 'common sense' and the foundation of our free will. We can relate 'intuition' to (1) the degree of certainty associated with judgment of a selected action course as the best and (2) the consequent degree of commitment to that action projection in the selected direction.

An unexpected outcome of behavioral analysis in terms of the psychonomic taxonomy was insight into the origin of negation and behavioral inhibition through socially contrived biofeedback in the learner, which led to an account of the origin of conscious choice and of the beginnings of consciousness.

We have now sketched the minimal required agenda of explanation of brain function as specified at the beginning of the first paper. Refutation of the foundation assumptions of the framework, or revision and expansion of the psychonomic theory in the light of new experiments to be formulated, is the task ahead. In any case, it is hoped that the systematic approach to theory generation attempted in these two papers will at least provide a stimulus for more coordinated and broadly based cognitive science research toward the ultimate goal of explaining the brain.

\section{REFERENCES}

ACredolo, L. P., \& GoodwyN, S. (1988). Symbolic gesturing in normal infants. Child Development, 59, 450-466.

Adler, L. E., Rose, G., \& Freedman, R. (1986). Neurophysiological studies of sensory gating in rats: Effects of amphetamine, phencyclidine and haloperidol. Biological Psychiatry, 21, 787-798.

Altshuller, G. S. (1984). Creativity as an exact science. The theory of the solution of inventive problems. (A. Williams, Trans.). New York: Gordon \& Breach Science Publishers.

Amaral, D. G., \& Sinnamon, H. M. (1977). The locus coeruleus: Neurobiology of a central norandrenergic nucleus. Progress in Neurobiology, 9, 147-196.

ANDERSEN, R. A. (1985). Encoding of spatial position by posterior parietal neurones. Science, $230,456$.
Appleton, T., Clifton, R., \& Goldberg, S. (1975). The development of behavioral competence in infancy. Review of Child Development, 4, 101-186

Aston-JONEs, G. (1985). Behavioral functions of locus coeruleus derived from cellular attributes. Physiological Psychology, 13, 118-126.

BEAR, M. F., Singer, W. (1986). Modulation of visual cortical plasticity by acetylcholine and noradrenaline. Nature, 320, 172-176.

Beauvols, M.-F. (1982). Optic aphasia: A process of interaction between vision and language. Philosophical Transactions of the Royal Society, London, Series B, 298, 35-47.

BLoom, L. (1975). Language development review. Review of Child Development Research, 4, 245-303.

Boorstin, D. J. (1986). The discoverers. Harmondsworth, England: Penguin.

Boyapati, J., \& Henry, G. H. (1985). The character and influence of the claustral pathway in the striate cortex of the cat. Experimental Brain Research, 61, 141-152.

Brinkworth, R. I., Lloyd, E. J., \& ANDrews, P. A. (1988). Brain Chemistry and central nervous system drugs. Natural Product Reports, 5, 363-386.

BUCY, P. C., \& KLÜVER, H. (1955). An anatomical investigation of the temporal lobe in the monkey (Macaca mulatta). Journal of Comparative Neurology, 103, 151-252.

BURTON, P. G. (1990). A search for explanation of the brain and learning. Elements of the psychonomic interface between psychology and neurophysiology: I. A cognitive approach to early learning. Psychobiology, 18, 119-161

Burton, P. G., Edwards, J. A. (in press). Pain and the regeneration of whole movement. Psychobiology.

CHURCH, A. (1956). Introduction to mathematical logic. Princeton, NJ: Princeton University Press.

ChURChLAND, P. S. (1986). Neurophilosophy: Towards a unified science of the mind/brain. Cambridge, MA: MIT Press.

Clynes, M. (1977). Sentics: The touch of emotions. London: Souvenir.

DARWIN, C. (1859/1984) The origin of species. Harmondsworth, UK: Penguin

Devine, E., Held, M., Vinson, J., \& Walsh, G. (Eds.) (1986). Thinkers of the 20ih century. London: Firethorn.

DEWEY, J. (1957). Human nature and conduct. New York: Random House. (Original work published 1922)

Englemann, S., Carnine, D. (1982). Theory of instruction: Principles and applications. New York: Irvington.

FAnN, K. T. (1970). C. S. Peirce's theory of abduction. The Hague: Martinus Nijhoff.

Feeney, D. M., Sutton, R. L., Boyeson, M. G., Hovda, D. A., \& DAIL, W. G. (1985). The locus coeruleus and cerebral metabolism: Recovery of function after cortical injury. Physiological Psychology, 13, 197-203.

Feigendaum, E., McCorduck, P. (1983). The fifth generation: Artificial intelligence and Japan's computer challenge to the rest of the world. Reading, MA: Addison-Wesley.

Felton, D., Hallman, H., Jonsson, G. (1982). Evidence for a neurotrophic role of norandrenergic neurons in the postnatal development of rat cerebral cortex. Journal of Neurocytology, 11, 119-135.

FETTERS, L., \& TODD, J. (1987). Quantitative assessments of infant reaching movements. Journal of Motor Behavior, 19, 147-166.

Foote, S. L., BLoom, F. E., \& Aston-Jones, G. (1983). Nucleus locus coeruleus: New evidence of anatomical and physiological specificity. Physiological Review, 63, 844-914.

Freeman, W. J. (1981). A physiological hypothesis of perception. Perspectives in Biology \& Medicine, 24, 561-592.

FreEMAN, W. J., \& SCHNEIDER, W. (1982). Changes in spatial patterns of rabbit olfactory EEG with conditioning to odors. Psychophysiology, 19, 44-56.

FrEGE, G. (1964). Basic laws of arithmetic (1893). [M. Furth, Trans.] Berkeley, CA: University of California Press.

Friedman, M. I. (1984). Teaching higher order thinking skills to gified students: A systematic approach. Springfield, IL: Thomas.

GALTON, F. (1870). Hereditary genius: An enquiry into its laws and consequences. New York: Appleton.

GARRETT, M. F. (1982). Production of speech: Observations from nor- 
mal and pathological language use. In A. W. Ellis (Ed.), Normality and pathology in cognitive functions (pp. 19-76). London: Academic Press.

Gazzaniga, M. (1986). Introduction. In G. Lynch (Ed.), Synapses, circuits and the beginnings of memory (p. viii). Cambridge, MA: MIT Press.

Georgopolous, A. P., Schwartz, A. B., \& Kettner, R. E. (1986) Neuronal population coding of movement direction. Science, 233, 1416-1419.

Geschwind, N. (1982). Disorders of attention: A frontier in neuropsychology. Philosophical Transactions of the Royal Society of London, Series $B, 298,173-185$

GLoor, P. (1978). Inputs and outputs of the amygdala: What is the amygdala trying to tell the rest of the brain? In K. E. Livingston \& O. Hornykiewicz (Eds.), Limbic mechanisms (pp. 189-209). New York: Plenum.

HALFORD, G. S. (1982). The development of thought. Hillsdale, NJ: Erlbaum.

HEBB, D. O. (1949). The organization of behavior. New York: Wiley.

Heimer, L. (1978). The olfactory cortex and the ventral striatum. In M. Livingstone \& H. Hornykiewicz (Eds.), Limbic mechanisms (pp. 95-187). New York: Plenum.

HewES, G. W. (1973). An explicit formulation of the relationship between tool-using, tool-making and the emergence of language. Visible Language, 7, 101-127.

HILL, J. C. (1986). A computational model which addresses errors of over-generalization and their subsequent disappearance in early childhood language acquisition. In 8th Annual Proceedings of the Cognitive Science Society (pp. 407-419). Hillsdale, NJ: Erlbaum.

Hill, J. C., \& ARBIB, M. A. (1984). Schemes, computation and language acquisition. Human Development, 27, 282-296.

HinToN, G. E. (1986). Learning distributed representations of concepts. In 8th Annual Proceedings of the Cognitive Science Society (pp. 112). Hillsdale, NJ: Erlbaum.

ILG, F. G., AMEs, L. B. (1955). Child behavior. New York: Dell. JoNes, E. G. (1985). The thalamus. New York: Plenum.

JunG, C. (1978). Man and his symbols. London: Picador. (Original work published 1964)

Karson, C. N., Goldberg, T. E., \& Leleszi, J. P. (1986). Increased blink rate in adolescent psychosis. Psychiatric Research, 17, 195-198.

Kasamatsu, T., Watabe, K., Scholler, E., \& Heggelund, P. (1983). Restoration of plasticity in cat visual cortex by electrical stimulation of the locus coeruleus. Society for Neuroscience Abstracts, 9, 911.

KAUER, J. S., \& ShEPHERD, G. M. (1977). Analysis of the onset phase of olfactory bulb unit responses to odor pulses in the salamander. Journal of Physiology, 272, 495-516.

Kelling, S. T., \& HALPERN, B. P. (1987). Taste judgments and gustatory stimulus duration: Simple taste reaction times. Chemical Senses, 12, 543-562.

KUnN, T. S. (1970). Structure of scientific revolutions (2nd ed.). Chicago: University of Chicago Press.

LANGER, S. K. (1967). An introduction to symbolic logic. New York: Dover.

LASHLEY, K. S. (1963). Brain mechanisms and intelligence. New York: Dover.

LEVINE, D. S. (1986). A neural network theory of frontal lobe function. In 8th Annual Proceedings of the Cognitive Science Society (pp. 716-727). Hillsdale, NJ: Erlbaum.

LEVY, W. B., \& DESMOND, N. L. (1985a). Associative potentiation/ depression in the hippocampal dentate gyrus. In G. Buzsaki \& C. H. Vanderwolf (Eds.), Electrical activity of the archicortex (pp. 359-373). Budapest: Akademiai Kiado.

LEVY, W. B., \& DESMOND, N. L. (1985b). Rules of elemental synaptic plasticity. In W. B. Levy, J. A. Anderson, \& S. Lehmkuhle (Eds.), Synaptic modification, neuron selectivity and nervous system organization (pp. 105-121). Hillsdale, NJ: Erlbaum.

libet, B., Gleason, C. A., Wright, E. W., \& Pearl, D. K. (1983). Time of conscious intention to act in relation to onset of cerebral ac- tivity (readiness-potential): The unconscious initiation of a freely voluntary act. Brain, 106, 623-646.

LLINAS, R. (1981). Electrophysiology of cerebellar networks. In V. B. Brooks (Ed.), Handbook of physiology (Sect. 1, Vol. II, Pt. 2, pp. 831-875). Baltimore: Williams \& Wilkins.

LURIA, A. R. (1961). The role of speech in the regulation of normal and abnormal behavior. New York: Pergamon.

LURIA, A. R. (1966). Higher cortical functions in man. London: Tavistock.

LURIA, A. R. (1973). The working brain. London: Penguin.

LURIA, A. R. (1976). The functional organization of the brain. In R. F. Thompson (Ed.), Progress in psychobiology (pp. 375-382). San Francisco: W. H. Freeman.

Marona-Lewicka, D., Michaluk, J., Antkiewicz-Michaluk, L., \& VeTALUNI, J. (1987). A comparison of locomotor responses to some psychotropic drugs and cerebral receptors in Acomys cahirinus and the laboratory mouse. Polish Journal of Pharmacology \& Pharmacy, 39, 293-302.

Matuin, M. (1983). Cognition. New York: Holt, Rinehart \& Winston.

McRae-Degueurce, A., Dennis, T., Leger, L., \& Scatton, B. (1985). Regulation of norandrenergic neuronal activity in the rat locus coeruleus by serotoninergic afferents. Physiological Psychology, 13, 188-196.

Meltzoff, A. N., \& Moore, M. K. (1977). Imitation of facial and manual gestures by human neonates. Science, 198, 75-78.

MEYER, B. (1988). Object-oriented software construction. Englewood Cliffs, NJ: Prentice-Hall.

Milner, B. (1959). The memory defect in bilateral hippocampal lesions. Psychiatric Research Reports, 11, 43-52.

MiLnER, B. (1982). Some cognitive effects of frontal lobe lesions in man. Philosophical Transactions of the Royal Society, London, Series $B, 298,211-226$.

Mixon, D., \& Burton, P. (1989). Faulty sensory perception. Direction, 1, 128-134.

MülLER, H. F. (1985). Prefrontal cortex dysfunction as a common factor in psychosis. Acta Psychiatrica Scandinavica, 71, 431-440.

NEWELL, G. (1973). Production systems: Models of control structures. In W. G. Chase (Ed.), Visual information processing. New York: Academic Press.

NewEll, G., \& SiMON, H. (1972). Human problem solving. Englewood Cliffs, NJ: Prentice-Hall.

Nissen, M. J., Knopman, D. S., \& SChacter, D. L. (1987). Neurochemical dissociation of memory systems. Neurology, 37, 789-794.

NoBLE, W. (1987). Perception and language: Towards a complete ecological psychology. In A. Costall, A. Still (Eds.), Cognitive psychology in question (pp. 128-141). Brighton, Sussex: Harvester Press.

Olpe, H.-R., SteinmanN, M. W., \& Jones, R. S. G. (1985). Electrophysiological perspectives on locus coeruleus: Its role in cognitive versus vegetative functions. Physiological Psychology, 13, 179-187.

Olson, C. R., \& Graybiel, A. M. (1980). Sensory maps in the claustrum of the cat. Nature, 288, 479-481.

O'Shea, L., SaAri, M., Pappas, B., Ings, R., \& Stange, K. (1968). Neonatal 6-hydroxydopamine attenuates the neural and behavioral effects of enriched rearing in the rat. European Journal of Pharmacology, 92, 43-47.

Parnevelus, J. G., \& Blue, M. E. (1982). The role of the norandrenergic system on the formation of synapses in the visual cortex of the rat. Developmental Brain Research, 3, 140-144

PAvlov, I. P. (1955). Selected works. Moscow: Foreign Languages Publishing House.

Perrett, D. I., Mistlin, A. J., \& Chiffy, A. J. (1987). Visual neurones responsive to faces. Trends in Neurosciences, 10, 358-364.

PETERS, A. M. (1983). The units of language acquisition. Cambridge: Cambridge University Press.

Pribram, K. H. (1987). The subdivisions of the frontal cortex revisited. In E. Perecman (Ed.), The frontal lobes revisited. New York: IRBN. Robbins, T. W., Everutt, B. J., Cole, B. J., ArCher, T., \& Mohammed, A. (1985). Functional hypothesis of the coeruleocortical 
norandrenergic projection: A review of recent experimentation and theory. Physiological Psychology, 13, 127-150.

Rosenberg, C. R., \& SeJNowsKI, T. J. (1986). The spacing effect on NETtalk, a massively parallel network. In 8th Annual Proceedings of the Cognitive Science Society (pp. 72-89). Hillsdale, NJ: Erlbaum.

ROTHWELL, J. C. (1987). Control of voluntary human movement. Beckenham, England: Croome Helm.

Rumelhart, D. E., \& McClelland, J. L. (1986). Parallel Distributed Processing: Explorations in the microstructure of cognition (Vols. 1-2). Cambridge, MA: MIT Press.

SARA, S. J. (1985). The locus coeruleus and cognitive function: Attempts to relate noradrenergic enhancement of signal/noise in the brain to behavior. Physiological Psychology, 13, 151-162.

SEABourne, P. L. (1975). An introduction to the Dienes mathematics programme. London: University of London Press.

SEGAL, M. (1985). Mechanisms of action of noradrenaline in the brain. Physiological Psychology, 13, 172-178.

SHEPHERD, G. M. (1979). The synaptic organization of the brain (2nd ed.). New York: Oxford University Press.

ShePHERD, G. M. (1983). Neurobiology. New York: Oxford University Press.

STEE LE, E. J. (1981). Somatic selection and adaptive evolution: On the inheritance of acquired characteristics (2nd ed.). Chicago: University of Chicago Press.

Stern, J. A., Walrath, L. A., Goldstein, R. G. (1984). The endogenous eyeblink. Psychophysiology, 21, 22-33.

Stevenson, S. B., Volkmann, F. C., Kelly, J. P., \& Riggs, L. A. (1986). Vision Research, 26, 1815-1824.

van Dongen, P. A. M. (1981). The central norandrenergic transmission and the locus coeruleus: A review of the data and their implications for neurotransmission and neuromodulation. Progress in Neurobiology, 16, 117-143.

Volkmann, F. C., Riggs, L. A., \& Moore, R. K. (1980). Eyeblinks and visual suppression. Science, 207, 900-902.

von HoFsten, C. (1979). Development of visually directed reaching: The approach phase. Journal of Human Movement Studies, 5, 160-178.

WATERHouse, B. D., WOODWARD, D. J. (1980). Interaction of norepinephrine with cerebrocortical activity evoked by stimulation of somatosensory afferent pathways in the rat. Experimental Neurology, 67, 11-34

WERNER, T. K., \& ShERRY, T. W. (1987). Behavioral feeding specialization in Pinaroloxias inornata, the "Darwin's Finch" of Cocos Island, Costa Rica. Proceedings of the National Academy of Sciences USA, 84, 5506-5510.

\section{NOTES}

1. The normal psychological use of the term 'inhibition' subsumes the more limited and technically specialized usage of this term in most of the present work.

2. Which, being disjoint, would automatically become dissociated through the subconscious processes of perceptual learning; see the first paper.

3. Although in the early stages of naming, selection of perceptually similar choices for children's names for objects (e.g., < cow > "moocow') is a common strategy to aid clarity of designation.

4. A developmental asymmetry in relation to learning of Type 1 and Type 2 objects arises from the necessity for a prerequisite sensorydissociation phase to support the limited-modality tracking over recurrences of Type 2 objects. The onset of Type 2 learning is thus (perhaps only slightly) deferred relative to Type 1 learning, which is directly available to the neonate.

5. One might speculate that the frustration of autism (see first paper) arises from an attention deficit, which gives rise to episodes that always appear too novel, or too dissimilar, to inhibit progressive and incremental learning within each domain.

6. "One of the most complex cutaneous reflexes is the scratch reflex of the spinal dog. Very light, moving stimulation of the hairs, mimicking the passage of an insect, can give rise to a vigorous scratching by the ipsilateral foot. The scratching is rhythmic, continues until the stimulus is removed and is directed quite specifically towards the site of stimulation. Despite their range, very little is known about the pathways which mediate these cutaneous effects" (Rothwell, 1987, p. 145).

7. See Meltzoff and Moore (1977) on imitation of facial and manual gestures by human neonates.

8. From at least 60 feeding attempts of each of 89 individual Cocos finches in a 3.4-hectare thicket, 62 were found to utilize just one of nine categories of feeding techniques for more than half of their feeding. These nonrandom feeding specializations seem to be acquired at least partially by juveniles observing at close quarters and imitating an adult feeding technique, through the fledglings foraging with their parents, or, outside the breeding season, in mixed species flocks (Werner \& Sherry, 1987).

9. "I will not attempt any definition of instinct. It would be easy to show that several distinct mental actions are commonly embraced by this term; but every one understands what is meant, when it is said that instinct impels the cuckoo to migrate and to lay her eggs in other birds' nests. An action, which we ourselves should require experience to enable us to perform, when performed by an animal, more especially a very young one, without any experience, and when performed by many individuals in the same way, without knowing for what purpose it is performed, is usually said to be instinctive. But I could show that none of these characters of instinct are universal. A little dose, as Pierre Huber puts it, of judgment or reason, often comes into play, even in animals very low in the scale of nature" (Darwin, 1859/1984, p. 234).

10. On the basis of single-unit recordings, Andersen (1985) reported a system of encoding spatial position relative to a head-centered coordinate space by monkey posterior parietal neurones, in which an eyeposition-dependent tuning for locations operates to control the average of three eye movements per second.

11. The distinction we make here is between concentrated attention across multiple episode cycles and primitive Type 2 object learning. which can be learned and mimicked on an intraepisode basis. However, the logical requirement for collective modal tracking is equivalent.

12. Nissen et al. reported that in humans, the administration of scopolamine, an anticholinergenic drug, reduced the ability to recall and recognize stimuli presented previously, abilities thought to require 'declarative' memory. In contrast, measures of 'procedural' memory were unaffected by scopolamine: performance on a serial reaction-time task incorporating a repeating stimulus and response sequence showed no difference in acquisition and retention of the sequence after scopolamine or saline. Evidently, the cholinergic system is required for declarative but not procedural memory.

13. Perrett, Mistlin, and Chiffy (1987) reported visual neurones of the superior temporal sulcus that are selectively responsive to faces Although some limited action (in saccadic movement during concentrated visual scrutiny) is probably necessary for activation of cells in the temporal lobe that are selectively responsive to 'grandmothers,' we can probably subsume this action within the basic automated operation of the visual system, rather than categorizing such action as part of the general kinaesthetic/proprioceptive modality for the present argument.

14. It becomes known to the learner only through personal awareness of recurrences by the learner.

15. No such 'automatic' labeling occurs internally.

16. This corresponds to the Type 1 'idea' of an action.

17. Which corresponds to the Type 2 'plan' of the action object.

18. The availability of stereotypically 'fused' speech remains in the automated but meaningless speech of aphasics.

19. Contrast the elaborate human cerebellum with that of the sheep, which exhibits a limited range of rather stereotyped actions in comparison to man.

20. This corresponds to the extracerebellar initiation of the action 'idea' and its automatic intracerebellar execution according to its cerebellar 'plan.'

21. It may be that a signal of completion of each movement-unit sequence within an action is necessary at the cortical level: "The projection from cerebellar nuclei to the thalamic nucleus $\mathrm{X}$ has only been discovered relatively recently. It forms the basis for a possible cerebellar 
influence on the activity of lateral area 6, the premotor area. At present, there have been no physiological studies of this pathway" (Rothwell, 1987 , p. 251).

22. Unilateral lesions of either or both structures have very little effect on gross movements.

23. Eighty percent of the time in the putamen.

24. "Timing of discharge of sensory inputs suggests that the main source of afferent information comes from the cerebral cortex. Putaminal neurones discharge 30 to $50 \mathrm{~ms}$ after onset of a torque pulse to the wrist. Globus pallidus neurones discharge slightly later with a latency of 40 to $60 \mathrm{~ms}$. This compares with $30 \mathrm{~ms}$ in the motor cortex" (Rothwell, 1987, p. 298).

25. This compares with $50 \%$ for motor cortex firing after EMG for similar tasks.

26. Probably through the agency of the acetylcholine neurotransmitter system.

27. We must admit that following dissociation and Type 2 learning of independent activities, should any two of these be initiated in coordination in an episode, the possibility will exist for activity-specific gating, rather than global gating of the whole percept-action system. Logically, we require global gating only during early learning; nothing in the logic prohibits the development of global activity that comprises multiply independent gating once the learner has matured.

28. We saw this in Kauer and Shepherd's (1977) data on olfaction in Figure 7 of the first paper.

29. Comparative studies of the laboratory and the spiny mouse (Acomys cahirinus) show differences in their motor-activity patterns and motor responses to morphine, apomorphine (a dopamine agonist), and clonidine (a noradrenaline agonist). The two species of mouse differed with respect to the distribution of cerebral $\alpha_{2}$-adrenergic receptors but showed no significant differences in other types of receptors $\left(\alpha_{1}\right.$-adrenergic, $\beta$-adrenergic, opiate $\mu$ and $\Delta$, or spiroperidol binding sites). Apparently, the high excitability of the Acomys is related to a deficit in the inhibitory noradrenergic transmission in the CNS.

30. See O'Shea, Saari, Pappas, Ings, and Stange (1968) on the attenuation by the noradrenaline antagonist 6-OHDA of the neural and behavioral effects of enriched rearing in the rat, which showed that the effects of drugs that influence locus coeruleus activity show up only if intoxication is combined with relevant experience, and which also showed that the locus coeruleus innervation of the cerebellum is implicated in motor learning. See also Amaral and Sinnamon (1977), Waterhouse and Woodward (1980), and van Dongen (1981), who have proposed noradrenaline as a regulator of cerebral 'tone' or 'signal-tonoise' ratio; Parnevelus and Blue (1982) on the role of the noradrenergic system in synapse formation in the visual cortex of the rat; Kasamatsu, Watabe, Scholler, and Heggelund (1983) on the restoration of plasticity in the cat visual cortex by electrical stimulation of the locus coeruleus; Segal (1985), who proposed a mechanism of action of noradrenaline in which it acts to suppress spontaneous activity but enhance evoked responses; Foote, Bloom, and Aston-Jones (1983) on locus coeruleus specificity; McRae-Deguerce, Dennis, Leger, and Scatton (1985) on regulation of norandrenergic effects by seretonergic afferents; Olpe, Steinmann, and Jones (1985) on the cognitive and the vegetative role of noradrenaline and its capacity to modify the effects of other transmitters-either reducing or potentiating their effects; Aston-Jones (1985) on the phasic activity of the locus coeruleus in the control of vigilance and the initiation of adaptive behavioral responses; Robbins, Everitt, Cole, Archer, and Mohammed (1985) on the complementary but distinctive role of noradrenaline and acetylcholine in the cortex; Sara (1985) on the functional recovery of rats after lesions to deplete noradrenaline; Feeney, Sutton, Boyeson, Hovda, and Dail (1985) on the role of noradrenaline in neuronal development and plasticity; and Felton, Hallman, and Jonsson (1982) on the neurotrophic role of noradrenaline in postnatal development of the rat cerebral cortex.

31. Bilateral removal of the complete temporal lobes in higher mammals leads to (1) overattentiveness (the animals are restless; they have an urge to orient toward or respond to all stimuli), (2) hyperorality (the animals compulsively examine all objects by putting them in their mouths), (3) psychic blindness (the animals see but do not understand; they indiscriminately approach and examine objects even though they are harmful, such as a lighted match), (4) sexual hyperactivity (the animals increase their sexual activity, even indiscriminately toward in- animate objects), and (5) emotional changes (previously wild and aggressive monkeys are rendered tame and placid, and can be handled easily) (Bucy \& Klüver, 1955; Shepherd, 1983, p. 536).

32. The interested reader may wish to follow up the broad amygdala story by referring to Gloor (1978), Shepherd (1983, especially Figures 29.9-10), and Brinkworth, Lloyd, and Andrews (1988, especially Figure 2 ).

33. Plasticity in the development of the visual system (Bear \& Singer, 1986) is known to be associated with the concerted action of two neurotransmitters.

34. Or better, with variable regard.

35. Most likely the amygdala operating in conjunction with the hippocampus.

36. Both the high-frequency eye tremor and the saccade are here assumed to be necessary supports to the basic processes of visual feature detection, so neither can be attributed such a higher role. We do not require full suppression of vision as the flag, but at least some remnant in a mature learner of the normal blink.

37. The blink is inherently sensitive to polymodal stimuli; it recurs in the human auditory startle reflex, the timing of which is modified by prior application of stimuli in other modalities (e.g., touch or vision).

38. See Karson, Goldberg, and Leleszi (1986) on increased blink rate in adolescent psychosis (7-25/min in psychotics compared to $4-16 / \mathrm{min}$ in controls). Blink-rate abnormalities have been noted in autistic (elevated blink rates) and mentally retarded children.

39. 'Cognitive load' and 'interest value' affect blink rate.

40. See Volkmann, Riggs, and Moore (1980) and Stevenson, Volkmann, Kelly, and Riggs (1986) on human visual suppression.

41. Age alters reflex blinking rate with stimulus duration.

42. Inhibition of the eyeblink reflex can occur in the human infant.

43. Libet et al. define two types of voluntary act. In Type I (where an experience of preplanning occurs), RP precedes $W$ by $800 \mathrm{msec}$ on average, and in Type II ('spontaneous' or 'capricious' acts), RP precedes $\mathrm{W}$ by $350 \mathrm{msec}$ on average, $150 \mathrm{msec}$ at minimum. In acquiring these measures, the subjects were asked not to blink from the time the CRO (cathode ray oscilliscope) spot started revolving until after the event.

44. Through subtle body language, which is predominantly nonverbal if verbal objects are involved in the to-be-made association.

45. From this we discern major practical significance (to later learning) of the social and cultural activities associated with the early teaching of 'number' ideas.

46. This may be exemplified by not trying to anticipate what one 'thinks' until one has heard what one is saying or seen what one is writing.

47. "I knew they couldn't study properly with the music blaring"unless that's the only way they ever study!

48. It was AD 1330 before an hour took on a precise year-round meaning of $1 / 24$ th of a day.

49. The development of the psychonomic theory over the last 4 years has been an attempt at this level of abstraction. The psychonomic framework has been developed from a broad basis of circumstantial evidence drawn from many relevant disciplines. The development of this framework is exemplary of the process that $\mathrm{C}$. S. Peirce described as abduction, as hypothesis formation (Fann, 1970), and for this reason I have presented most of the argument in the original order of its development.

The project began in mid-1985 after completion of NUMBER DETECTIVE. A first draft of the first paper was completed prior to my visits during August-September 1986 to Professors Ian Darian-Smith (Department of Physiology, University of Melbourne), Karl Pribram (Department of Psychology, Stanford University), Michael Arbib (Department of Computer Science, University of Massachussetts), Charles Greer (Department of Anatomy and Physiology, Yale University), Pierre Gloor (Montreal Neurological Institute), Jean-Claude Brief (Faculty of Education, University of Quebec), Herbert Müller (Douglas Psychiatric Hospital, Montreal), Michael Gazzaniga (Cognitive Neuroscience Unit, New York), Julian Jaynes (Department of Psychology, Princeton University), and William Levy (University of Virginia Medical School). In each case, stimulating discussion and guidance is gratefully acknowledged. A second draft of the first paper and the initial segments of the present paper were completed in November 1987, and were presented at a number of seminars in Australia, Japan, and Europe in 1988 and early 1989. 
I have sought simple and clear verbal description of each element of the interface between the neurophysiological 'wetware' and the psychological appearance of our exhibited behavior. Because this is a difficult task when presenting novel ideas, I have attempted to support the text with clear designation and visual symbolism to represent the conceptual basis of the psychonomic framework, using diagrams of variously one-dimensional (Type 1 symbolism for declarative knowledge), twodimensional (Type 1/Type 2 symbolism for procedural knowledge), and three-dimensional (Type 1/Type 2/intensional symbolism for conceptual or abstract metaknowledge).

50. I extend sincere thanks to Chris Peacock and to the Psychobiology editorial staff for their assistance in this project.

\section{APPENDIX}

\section{The Five Assumptions of the Psychonomic Theory of the Brain's Operation}

\section{Assumption 1: Universal Computational Logic of Learning}

In seeking to articulate a theoretical description of the brain's operation, one must first recognize that learning can be categorized broadly into three ontogenetic types: perceptual learning, motor-skill learning, and conceptual learning. There seems to be no reason to begin with a requirement that each type of learning requires a fundamentally different computational logic, even if different neurophysiological infrastructures were to be implicated in the different learning types. Accordingly, we seek a parsimonious logic, a universal learning logic that can support each kind of learning. Since the content of learning is different in each learning type, we could admit some data structure differences while requiring the same general logic of learning for each.

\section{Assumption 2: The Possibility of Autonomous Learning}

Despite the socially and culturally structured environment in which infants are nurtured, we assume that autonomous learning is logically possible, that it actually occurs, and that learning is possible even when the learner is confronted with a totally novel environment. That is, we assume that the brain offers the potential to support learning de novo. The logical possibility of learning by an autonomous neophyte is assumed so that no knowledge is required to be innate: we begin with the tabula rasa. Continued learning in any domain is assumed to accelerate and focus this intrinsic learning process for reasons other than a change in the basic computational logic.

\section{Assumption 3: Explicitly Discrete Cerebral Processing in Episodes}

Despite the continuity of our conscious experience, and in the face of the apparent cyclical continuity of psychophysiological indicators such as EEG waves, we assume that no evidence rules out logically and temporally discrete processing in various functional units of the brain, particularly below the cortex. In addition, we assume that a physiological gating of central cognitive system processing is possible (Adler et al., 1986). Logically discrete, physiologically gated cerebral processing is assumed in order to provide a basis for temporally quantized episodic processing.

\section{Assumption 4: Episode Processing is Ultimately Selective but Begins Data-Driven and Subconscious}

The deferred onset of behavior interpreted as conscious choice is assumed to imply that early learning is subconscious and data-driven. The data-driven character of episodic processing implies an irregular temporal gating, especially once processing becomes selective.

Data-driven episode processing is characterized by a requirement that, in the acquisition of each new storable episode, a minimal degree of novelty in content compared to currently recallable (i.e., relevant) episodes be attained as the threshold condition of episode gating. Furthermore, we assume that this mode of learning remains the principal mode of learning even after the onset of conscious behavior. In adults, this mode of learning is characterized by the ordinary, everyday 'ticking-over' behavior associated with non-problem-solving activities (i.e., the preponderance of the waking hours). Consciousness is associated with selecting alternative domains as the ticking-over domain, with retrospective 'narratization' of behavior and with communication based upon prior intraepisodic 'snap' judgments that are reached by an inherently subconscious process. In any domain, consciously rational behavior is rare because (1) any prospective character to thought depends upon extensive prior learning within that domain (and others), and (2) the 'exit' from the normal ticking-over mode to the problem-solving mode is infrequently prompted.

\section{Assumption 5: Direct Perception is the Basis of Learning}

Phylogenetic evolution has provided an environmentally adapted selective perceptual apparatus for each organism: independent information channels from each such source are the basis of all learning. Irrespective of the distributed preprocessing that is necessary to yield the content of each information channel and whatever the path of neuronal communication to the central cognitive system (CCS), we need assume only that recurrence of the same stimulus provides perceptual information that can be registered at the CCS through the same information paths, so that it may be perceived the same. This corresponds to a direct indexing of perceptual information to the CCS. No confounding of information in independent channels is required; indeed, it is contraindicated by the insulation of independent communication channels to (and from) the CCS. For this reason, a vector metaphor is adopted to describe perceptual input registered at the CCS.

In principle, information channels may be highly derivative of sensory activation in the peripheral apparatus or even of the primary sensory-cortex activation. Thus, information channels may range in complexity from 'slanted-edge specific' to putative 'grandmother-specific' cells in vision and need not necessarily be based in a single modality.

\section{POSTSCRIPT: DISSOCIATIVE LEARNING, SYMBOLISM, AND DEGREES OF ABSTRACTION}

In the cognitive approach to learning, objectification (internal object intension formation) arising from recurring episodes containing a perceptual object initially proceeds via dissociation of the object from its background. This is the first generation of dissociative learning, which applies to Type 1 objects, the things in the environment. Figure 15 illustrates one aspect of the process of objectification through experience with successive instances of similar Type 2 objects, in the creation of the possibility of anticipation of the outcome of a frequently experienced motor transform (which may be either internally generated or effected by an external agent). Objectification over primitive Type 2 objects allows for the possibility of virnual or imagined transforms, the outcome of which can be anticipated 
without the need to explicitly undertake the transform. By abstracting over time on the basis of recurrences of the transform, one gains the possibility of assessing the expected outcome of one or more familiar motor transforms ahead of actual implementation. This is the second generation of objective dissociation on the basis of experience. The availability of such anticipation corresponds broadly to Piaget's 'operational stage' of child development, but some forms of anticipation are exhibited by human infants as early as 3 months of age.

Elementary conceptual learning attributes perceptually disjoint symbolic labels to physical objects (nouns) and to objectified actions (verbs), and in early language acquisition we progressively learn to dissociate the use of these symbols from the fundamental referents [in the physical objects or in actual transforms (i.e., the actual experience)] upon which, for each of us, each language element is based.

The attribution of labels to objects (and their attributesadjectives-learned by experience) and to actions (and their attributes-adverbs-learned by experience) is associative learning, but this is merely an enabling transition that affords the possibility of language and thought to progress to a symbolic basis ever more divorced, but never fully abstracted, from actual referents.

The meaningfulness of each verbally denoted concept depends entirely on the variety of its extensional episodic base, which, with increasing experience, generates an ever-broader network of connectivity of the concept to other object intensions of our memory. And as is so often the case, here also it is the counterexample (e.g., Beauvois, 1982) which delivers most clarity to the idea intended.

The reality of the deep interconnectivity between a conceptual symbol and its actual referential base in episodes of experience and the constant instantaneous availability of crossreference through this network is proven by the distinguishing human capacity to both generate and appreciate humor. Machines will never be capable of intelligent, creative work without first being capable of generating humorous 'utterances.'

The general principles of personal learning through dissociation over experience naturally operate to encompass any observed experience of others that is relevant to a learning domain. With some difficult concepts, the progress toward abstraction of the concept is not so much a personal as a societal achievement, with the learning sometimes tortuous and taking many individual lifetimes. Boorstin (1986, pp. 1-78) provides an illuminating account of human progress in the dissociative learning and abstraction of time; the major steps of achieving a common definition of an hour ${ }^{48}$ and later subdivision of this unit into minutes and seconds requires the accumulation of much experience: "To use the sun's shadow at any place to define the hour according to Greenwich Mean Time requires a knowledge of astronomy, geography, mathematics and mechanics. Not until the sixteenth century could sundials be marked with these true hours" (Boorstin, 1986, p. 27).

Boorstin also recounts how exploration of the world was guided and constrained by the world maps available in each era. Symbolic representation of the domain has often played an important part in learning through the experience of others, in human anatomy as in geography. Leonardo da Vinci had warned against the delusive precision of verbal anatomy texts: "The more minute your description the more you will confuse the mind of the reader and the more you will lead him away from the knowledge of the thing described. It is necessary for you to represent and describe" (Boorstin, 1986, p. 379).

Dissociation (objectification) over procedures experienced is the goal of formal education, which is ultimately aimed at the objectification of symbolic procedures and the enabling of their production. The formal education process constitutes the basis of the third generation of dissociative learning each of us experiences.

Good computer-based examples of formal approaches to aid the comprehension and objectification of procedures are the children's programs LOGO, and Number Detective and Number Explorer from Spinifex Software, available for Apple II computers. These programs exemplify third-generation computing.

Abstraction to a higher degree again is involved in the constructive or creative use of symbolic procedures to produce new outcomes. This is exemplified by the disciplines of logic, mathematics, physics, chemistry, procedural computation, architecture, literary or musical production, and philosophical analysis, as well as in, say, the construction of a business plan. In computation, it is exemplified by the Apple Macintosh's 'point and click' computational environment as fourth-generation computing.

At the fifth level of dissociation, where successive symbolic procedures are further abstracted, we have symbolic logic, pure mathematics, literary criticism, the systematization of the basis of creativity in the patent literature (Altshuller, 1984), and logicbased fifth-generation computation (Feigenbaum \& McCorduck, 1983). This is the level of disciplinary-based theory formation, exemplified in descriptions of the 'scientific method.'

At a yet higher level of abstraction, we have dissociation across exemplars of formal theories. This level corresponds to 'scientific revolution' (see Kuhn, 1970), the progress of quantum mechanics, cosmology, and adaptive expert systems. All of these share the characteristic of incremental adaption to progressively changing external information environments; they go beyond the notion of static, delimited knowledge.

Adaptive expert systems, constituted from integrated rulebased and neural network elements that can 'learn' in response to examples, correspond to the sixth generation of computation. Such adaptive expert systems were just appearing as the 1980s closed. These systems for decision support progressively and dynamically adjust to changes in external context.

The seventh level of dissociation from the real world comprises transdisciplinary approaches to complex problems, as in the developing areas of environmental science, which are aimed at understanding the entirety of our interconnected ecological environment as we disturb it, and cognitive science, which is aimed at understanding the whole of the brain as we change it by learning. ${ }^{49}$

The computational correlate of the seventh degree of dissociation (seventh-generation computing) will comprise computerbased decision systems, which, like these two interdisciplinary academic areas and the brain itself, support (1) multiple independent decision-path analysis and (2) a dynamically restructuring capacity in the semantic base of decisions to provide experiencebased substructuring of semantic elements-that is, the capacity to explicitly learn and to learn to explain progressively more clearly on the basis of experience.

In this way, one can begin to sketch an intellectual road map through elements of our culture. It remains to be seen whether education or training procedures for more efficient yet effective vicarious learning can be developed so that people can become constructive and creative at yet a higher level of abstraction. It is hoped that progression to each successively higher level of competence in thought will enable better management of the uncertainty that engulfs us and will enhance the adaptive capacity of the species. ${ }^{50}$

(Manuscript received October 1988; revision accepted for publication October 1989.) 